\title{
Prediction of ALK mutations mediating ALK-TKIs resistance and drug re-purposing to overcome the resistance
}

\section{$\operatorname{AUTHOR}(\mathrm{S}):$}

Okada, Koutaroh; Araki, Mitsugu; Sakashita, Takuya; Ma, Biao; Kanada, Ryo; Yanagitani, Noriko; Horiike, Atsushi; ... Okuno, Yasushi; Fujita, Naoya; Katayama, Ryohei

\section{CITATION:}

Okada, Koutaroh ...[et al]. Prediction of ALK mutations mediating ALK-TKIs resistance and drug re-purposing to overcome the resistance. EBioMedicine 2019, 41: 105-119

\section{ISSUE DATE:}

2019-03

\section{URL:}

http://hdl.handle.net/2433/255588

\section{RIGHT:}

(C) 2019 The Authors. Published by Elsevier B.V. This is an open access article under the CC BY-NC-ND license

(http://creativecommons.org/licenses/by-nc-nd/4.0/). 


\title{
Prediction of ALK mutations mediating ALK-TKIs resistance and drug re-purposing to overcome the resistance
}

\author{
Koutaroh Okada a,b,1, Mitsugu Araki c,d,1, Takuya Sakashita a , Biao Ma e, Ryo Kanada ${ }^{\text {f }}$, Noriko Yanagitani ${ }^{\mathrm{g}}$, \\ Atsushi Horiike ${ }^{\mathrm{g}}$, Sumie Koike ${ }^{\mathrm{a}}$, Tomoko Oh-hara ${ }^{\mathrm{a}}$, Kana Watanabe ${ }^{\mathrm{h}}$, Keiichi Tamai ${ }^{\mathrm{i}}$, Makoto Maemondo ${ }^{\mathrm{h}}$, \\ Makoto Nishio $^{g}$, Takeshi Ishikawa ${ }^{\mathrm{j}}$, Yasushi Okuno ${ }^{\mathrm{c}, \mathrm{d}}$, Naoya Fujita ${ }^{\mathrm{a}, \mathrm{b}}$, Ryohei Katayama ${ }^{\mathrm{a}, *}$ \\ a Division of Experimental Chemotherapy, Cancer Chemotherapy Center, Japanese Foundation for Cancer Research, Tokyo, Japan \\ b Department of Computational Biology and Medical Sciences, Graduate School of Frontier Sciences, The University of Tokyo, Tokyo, Japan \\ c RIKEN Advanced Institute for Computational Science, 7-1-26 Minatojima-Minamimachi, Chuo-ku, Kobe, Hyogo 650-0047, Japan \\ ' Graduate School of Medicine, Kyoto University, 53 Shogoin-Kawaharacho, Sakyo-ku, Kyoto 606-8507, Japan

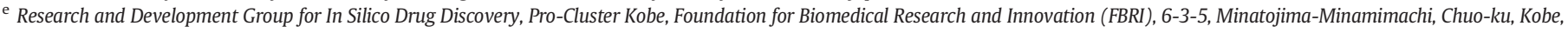 \\ Hyogo 650-0047, Japan \\ ${ }^{\mathrm{f}}$ RIKEN Compass to Healthy Life Research Complex Program, 6-3-5, Minatojima-Minamimachi, Chuo-ku, Kobe, Hyogo 650-0047, Japan \\ g Department of Thoracic Medical Oncology, The Cancer Institute Hospital, Japanese Foundation for Cancer Research, Tokyo, Japan \\ ${ }^{\text {h }}$ Department of Respiratory Medicine, Miyagi Cancer Center, Miyagi, Japan \\ i Division of Cancer Stem Cell, Miyagi Cancer Center Research Institute, Miyagi, Japan \\ j Department of Molecular Microbiology and Immunology, Graduate School of Biomedical Sciences, Nagasaki University, 1-12-4 Sakamoto, Nagasaki 852-8523, Japan
}

\section{A R T I C L E I N F O}

\section{Article history:}

Received 8 August 2018

Received in revised form 8 January 2019

Accepted 8 January 2019

Available online 17 January 2019

\section{Keywords:}

ALK-rearranged lung cancer

Resistant mutation

Computational simulation

MP-CAFEE

Compound mutation

Quantum chemistry

\begin{abstract}
A B S T R A C T
Background: Alectinib has shown a greater efficacy to $A L K$-rearranged non-small-cell lung cancers in first-line setting; however, most patients relapse due to acquired resistance, such as secondary mutations in ALK including I1171N and G1202R. Although ceritinib or lorlatinib was shown to be effective to these resistant mutants, further resistance often emerges due to ALK-compound mutations in relapse patients following the use of ceritinib or lorlatinib. However, the drug for overcoming resistance has not been established yet.

Methods: We established lorlatinib-resistant cells harboring ALK-I1171N or -G1202R compound mutations by performing ENU mutagenesis screening or using an in vivo mouse model. We performed drug screening to overcome the lorlatinib-resistant ALK-compound mutations. To evaluate these resistances in silico, we developed a modified computational molecular dynamic simulation (MP-CAFEE).

Findings: We identified 14 lorlatinib-resistant ALK-compound mutants, including several mutants that were recently discovered in lorlatinib-resistant patients. Some of these compound mutants were found to be sensitive to early generation ALK-TKIs and several BCR-ABL inhibitors. Using our original computational simulation, we succeeded in demonstrating a clear linear correlation between binding free energy and in vitro experimental $\mathrm{IC}_{50}$ value of several ALK-TKIs to single- or compound-mutated EML4-ALK expressing Ba/F3 cells and in recapitulating the tendency of the binding affinity reduction by double mutations found in this study. Computational simulation revealed that ALK-L1256F single mutant conferred resistance to lorlatinib but increased the sensitivity to alectinib.

Interpretation: We discovered lorlatinib-resistant multiple ALK-compound mutations and an L1256F single mutation as well as the potential therapeutic strategies for these ALK mutations. Our original computational simulation to calculate the binding affinity may be applicable for predicting resistant mutations and for overcoming drug resistance in silico.

Fund: This work was mainly supported by MEXT/JSPS KAKENHI Grants and AMED Grants.
\end{abstract}

(c) 2019 The Authors. Published by Elsevier B.V. This is an open access article under the CC BY-NC-ND license (http:// creativecommons.org/licenses/by-nc-nd/4.0/).

\footnotetext{
* Corresponding author.

E-mail address: ryohei.katayama@jfcr.or.jp (R. Katayama).

1 These authors contributed equally to this work.
}

\section{Introduction}

In 2007, Soda and his colleagues found an echinoderm microtubuleassociated protein-like 4 (EML4)-ALK fusion gene from non-small-cell lung cancers (NSCLCs) [1]. ALK-rearranged NSCLCs account for 3\%-5\% 


\section{Research in context}

\section{Evidence before this study}

The first-line ALK-TKI alectinib treatment is an effective therapeutic strategy against $A L K$-rearranged non-small-cell lung cancers. ALK-I1117N or -G1202R mutations positive tumors have been often found in alectinib-resistant cancers, but these tumors are sensitive to ceritinib or lorlatinib. However, further resistance often emerges due to ALK-compound mutations in relapse patients following the use of ceritinib or lorlatinib.

\section{Added value of this study}

This study provides ALK-compound mutations conferring lorlatinib or ceritinib resistance. Moreover, we found that the some of these compound mutations were sensitive to clinically approved ALK-TKIs or several BCR-ABL inhibitors. Additionally, we successfully developed a computational simulation to estimate accurately the affinity of ALK-TKIs to each single resistant ALK mutants and to various compound mutations. Furthermore, we identified lorlatinib resistant ALK-L1256F single mutation in addition to ALK compound mutations.

\section{Implications of all the available evidence}

Our results provide potential therapeutic strategies against some lorlatinib- or ceritinib-resistant compound mutations, and support the usefulness of our newly developed in silico computational simulation to predict resistance conferred by kinase mutations and effective candidate drugs.

of lung adenocarcinoma in East Asia and for 3\%-6\% in US/Europe [2]. These ALK fusion proteins can induce the constitutive activation of the ALK-tyrosine kinase via the oligomerization of domains such as the coiled-coil domain of fusion partner. As a result, ALK downstream pathways, including the PI3K-AKT-mTOR, RAS-MAPK-ERK, or JAK-STAT pathways, are constitutively activated [3,4]. The ALK-tyrosine kinase inhibitor (TKI) crizotinib was first applied for the treatment of EML4ALK-positive NSCLC based on the improved clinical outcomes of using crizotinib in clinical trials $[5,6]$. Although crizotinib has been used for treatment in both first- and second-line settings, a phase III clinical trial comparing crizotinib and alectinib as a first-line treatment for ALK-rearranged NSCLC, so called ALEX and J-ALEX studies, have demonstrated that alectinib was markedly superior to crizotinib in extending PFS and has a favorable safety profile $[7,8]$. Based on these studies, alectinib is now one of the first-line treatment options for ALKpositive NSCLCS.

Most patients respond to alectinib; unfortunately, patients inevitably relapse within several years due to acquired resistances $[9,10]$. Among the alectinib-resistant mechanisms, secondary point mutations, such as G1202R or I1171N, in the ALK kinase domain are very frequent [9]. The $11171 \mathrm{~N}$ mutation is reported to be sensitive to the secondgeneration ALK inhibitor ceritinib in vitro and in patients [10]. However, the G1202R mutation is resistant to first- and second-generation ALK inhibitors (crizotinib, alectinib, and ceritinib). The other secondgeneration ALK-TKI brigatinib was shown to be active against the G1202R mutant in vitro and in vivo. However, it was noted that G1202R mutation was discovered in about 50\% relapse patients following the use of brigatinib. Currently, the next-generation ALK inhibitor lorlatinib (PF-06463922) has been shown to be active against almost all of the previously identified ALK-TKI resistance mutations, including G1202R [11]. However, sequential ALK-TKI treatment has been reported to induce complex resistance mechanisms, including compound mutations. The ALK-E1210K + D1203N double mutation was discovered from a brigatinib-relapsed specimen of an $A L K$-rearranged lung cancer treated with first-line crizotinib. This double mutation was shown to be sensitive to lorlatinib in vitro [9]. Currently, although multiple ALKcompound mutants have been identified from lorlatinib sequential therapy resistant patients [12,13], the overcoming drugs against most of these mutants have not yet been clarified. To identify the lorlatinib or ceritinib resistance mechanisms in the ALK-G1202R or I1171N mutation-positive cancers, we performed N-ethyl-N-nitrosourea (ENU) mutagenesis screening and established a lorlatinib-resistant tumor using the EML4-ALK-G1202R mutation harboring ALK-positive lung cancer cells in an in vivo mouse model. Molecular dynamic (MD) free energy simulation by the use of MP-CAFEE [14] successfully showed a clear linear correlation between experimental IC $\mathrm{C}_{50}$ values of each ALKTKI obtained using Ba/F3 cells expressing single- or compound-mutated EML4-ALK and the binding affinities of the ALK-TKI to the corresponding mutants. In addition, fragment molecular orbital (FMO) method [15] precisely quantified a marginal difference in the ALK-drug (alectinib) interaction among ALK mutants (I1171N, I1171N + L1256F, and L1256F), which could not be detected by the conventional MD simulation. Furthermore, we newly found and confirmed that L1256F single mutation confers marked resistance to lorlatinib but is extremely sensitive to alectinib. For a lorlatinib-resistant G1202R + L1196M double mutation, which is highly resistant to all ALK-TKIs, we found potential agents to suppress the resistant double mutation using high throughput drug screening. Our study models the possible lorlatinib-resistant compound mutations and shows potential therapeutic strategies to suppress this resistance.

\section{Materials and methods}

\subsection{Cell lines and reagents}

Human embryonic kidney cells, 293FT cells (Invitrogen), were cultured with Dulbecco's Modified Eagle Medium high glucose (DMEM) supplemented with $10 \%$ fetal bovine serum and kanamycin (Meiji Seika Pharma, $250 \mathrm{mg} / \mathrm{ml}$ ). And murine bone marrow derived pro-B cells, Ba/F3 cells, were cultured in DMEM low glucose supplemented with $10 \%$ fetal bovine serum, kanamycin and $0.5 \mathrm{ng} / \mathrm{ml}$ of interleukin3 (IL-3). The cells were infected with retrovirus replicated in 293FT cells by transforming them with paging plasmids (pLenti), which contained rearranged cDNA regions encoding EML4-ALK variant 1 and either wild-type or different resistance mutations (lorlatinib, ceritinib or alectinib). The pENTR/D-TOPO vector (Thermo Fisher Scientific) was used to clone the different cDNA regions by utilizing LR clonase II reactions; cells were selected with blastcidin $(7 \mu \mathrm{g} / \mathrm{ml})$ for 1 week. After the selected cells grew, they were cultured in DMEM without IL3. Alectinib- or ceritinib-resistant EML4-ALK (variants 3)-G1202R mutation-expressing patient-derived cell line JFCR-041-2 cells were cultured in StemPro hESC medium (Thermo Fisher Scientific) supplemented with $1 \times$ Antibiotic-Antimycotic Mixed Stock Solution (Nacalai tesque) and Y27632 (10 $\mu \mathrm{M})$. Alectinib-resistant EML4-ALK (variants 3)-I1171N mutation-expressing patient-derived cell line JFCR-043-2 cells were cultured in media in which RPMI1640 (Thermo Fisher Scientific) and Ham's F-12 (Nacalai tesque) were mixed in equal proportions, supplemented with $10 \%$ FBS and $1 \times$ Antibiotic-Antimycotic.

Crizotinib (PF-02341066), lorlatinib (PF-06463922) or brigatinib (AP26113) were obtained from Shanghai Biochempartner. Alectinib (CH5424802) and ceritinib (LDK-378) was purchased from ActiveBiochem. 17-AAG was purchased from LC Laboratories. AG-957 was purchased from the Cayman Chemical Company. Adaphostin was purchased from SIGMA. Brigatinib was dissolved in ethanol for cell culture experiments. Other compounds were dissolved in dimethyl sulfoxide (DMSO) for cell culture. 


\subsection{Antibodies and immunoblotting}

$\mathrm{Ba} / \mathrm{F} 3$ cells $\left(1 \times 10^{6}\right)$ were seeded into 12 -well plates and treated with different drugs for $3 \mathrm{~h}$. For patient-derived cell lines, $3 \times 10^{5}$ to 1 $\times 10^{6}$ cells were seeded into collagen coated 6 -well plates. After 48 to $72 \mathrm{~h}$, the cells were treated with the indicated ALK inhibitors for $3 \mathrm{~h}$. Cells were suspended in lysis buffer containing $0.1 \mathrm{M}$ Tris ( $\mathrm{pH} 7.5$ ), $10 \%$ glycerol, and $1 \% \mathrm{SDS}$ and boiled at $100{ }^{\circ} \mathrm{C}$ for $5 \mathrm{~min}$. The protein concentrations were measured with a BCA Protein assay Kit (Thermo Fischer Scientific). The lysates were adjusted to $1 \mu \mathrm{g} / \mu \mathrm{g}$ with lysis buffer, and added $20 \%$ volume of sample buffer containing $0.65 \mathrm{M}$ Tris (pH 6.8), 20\% 2-mercaptoethanol, 10\% glycerol, 3\% SDS, and $0.01 \%$ bromophenol blue. $10 \mu \mathrm{g}$ of each sample were electrophoresed by sodium dodecyl sulfate-polyacrylamide gel electrophoresis (SDSPAGE), and immunoblotted with the antibodies against total ALK (\#3633S, Cell Signaling Technology), phospho-ALK (Y1604; \#3341S, Y1282/83; \#9687S, Cell Signaling), total S6 Ribosomal Protein (\#2217S, Cell Signaling Technology), total p42/44 ERK/MAPK (\#9102S, Cell Signaling Technology), phospho- p42/44 ERK/MAPK (\#9101S, Cell Signaling Technology), total AKT (\#4691S, Cell Signaling Technology), phospho-AKT (S473; \#4060S, Cell Signaling Technology), total STAT3 (\#4904S, Cell Signaling Technology), phospho-STAT3 (\#9145S, Cell Signaling Technology), $\beta$-Actin (SIGMA) or GAPDH (MAB374, Millipore).

\subsection{In vivo study of lorlatinib, ceritinib, or alectinib}

All in vivo studies were conducted through the Institutional Animal Care and Use Committee-approved animal protocols according to the institutional guidelines. The tumor pieces (approximately $8 \mathrm{~mm}^{3}$ ) of the JFCR-043-2 or JFCR-041-2 cells were subcutaneously transplanted into BALB-c $n u / n u$ mice (Charles River). After the tumor volumes reached approximately $200 \mathrm{~mm}^{3}$, the mice were randomized into vehicle control, lorlatinib, ceritinib, or alectinib groups (with $n=6$ in each group). For the mice with the JFCR-043-2-transplanted cells, we used vehicle control, $60 \mathrm{mg} / \mathrm{kg}$ alectinib, $60 \mathrm{mg} / \mathrm{kg}$ ceritinib, or $25 \mathrm{mg} / \mathrm{kg}$ lorlatinib. On the other hand, for the mice with the JFCR-041-2- and JFCR-041-2 LorR-transplanted cells, we used vehicle control, alectinib $50 \mathrm{mg} / \mathrm{kg}$, lorlatinib $10 \mathrm{mg} / \mathrm{kg}$, or brigatinib $50 \mathrm{mg} / \mathrm{kg}$. The mice were continuously treated with these agents once a day using oral gavage for 5-6 days/week. The tumor volumes and body weights were measured twice weekly. Relative tumor size was calculated by dividing the tumor size of each day by that of the treatment initiation day. When the tumor size exceeded approximately $1000 \mathrm{~mm}^{3}$, the mice were euthanized.

For the establishment of lorlatinib-resistant JFCR-041-2 LorR tumors in vivo, the JFCR-041-2 cells-bearing BALB-c nu/nu mice were treated with lorlatinib $10 \mathrm{mg} / \mathrm{kg}$. Once the JFCR-041-2 tumor grew under the lorlatinib $10 \mathrm{mg} / \mathrm{kg}$ treatment, the tumor pieces were re-transplanted into nude mice, and these were then treated with lorlatinib $30 \mathrm{mg} / \mathrm{kg}$; the dose was gradually increased up to $50 \mathrm{mg} / \mathrm{kg}$. Total cDNA was prepared from the total RNA purified from the resultant lorlatinibresistant tumors. Further, the ALK kinase domains were sequenced with Sanger Sequence.

\subsection{ENU mutagenesis screening}

Single clonal cells $\left(1 \times 10^{6}\right.$ cells $\left./ \mathrm{ml}\right)$ were exposed to N-ethyl-Nnitrosourea (ENU) from SIGMA at concentrations of $100 \mu \mathrm{g} / \mathrm{ml}$ for 24-28 h. Then, the cells were washed with $50 \mathrm{ml}$ of PBS and cultured in fresh DMEM for 24-48 h. Further, the cells with randomly induced mutations were seeded in 96-well plates (ALK-WT\#13; $1 \times 10^{5}$ cells, G1202R\#30 or \#43; $5 \times 10^{4}$ cells, or I1171N\#12; $3 \times 10^{5}$ cells in each well). The cells were cultured with lorlatinib $(300,600$, or $1000 \mathrm{nM})$, ceritinib $(300,600$, or $1000 \mathrm{nM})$, or alectinib $(100,500$, or $1000 \mathrm{nM})$ for 2-4 weeks. The resistant clonal cells were picked and expanded.
Then, genomic DNA was prepared from these resistant cells by lysing them with proteinase $\mathrm{K}$ buffer. The regions of DNA encoding the ALK kinase domain amplified using KOD-Plus-Neo (TOYOBO) polymerase were sequenced.

\subsection{Cell viability assays}

The cells were seeded in triplicate into 96 well plates and treated with serial dilutions of different inhibitors. For the Ba/F3 cells, 2000 cells were plated into 96 well plates in triplicates and cultured in the media containing different drugs for $72 \mathrm{~h}$. After incubation for $72 \mathrm{~h}$, the cells were incubated with CellTiter-Glo reagent (Promega) for $10 \mathrm{~min}$, and luminescence was measured using TriStar LB941 (Berthold Technologies). To analyze the data, the GraphPad Prism version 5.0 (GraphPad software) was used. The $\mathrm{IC}_{50}$ values were determined using a nonlinear regression model with a sigmoidal dose response in GraphPad.

\subsection{In vitro kinase assay of ALK protein and ALK-inhibitors}

The recombinant proteins of the kinase domain of wild-type ALK and ALK-L1256F were purchased from Signal Chem. Appropriate amounts of target proteins were calculated as recommended by the ADP-Glo Assay manufacturer's protocol after incubating in 96-well half area white plates with serially diluted inhibitor over a 9-dose range ( $1 \mathrm{nM}-10 \mu \mathrm{M})$ for $10 \mathrm{~min}$ at the room temperature. ATP at concentrations of 10,30 , and $100 \mathrm{nM}$ was mixed with $100 \mu \mathrm{g} \mathrm{ml}^{-1}$ substrate and added to a kinase protein-inhibitor mixture, followed by incubation for $60 \mathrm{~min}$ at the room temperature. After the kinase reaction, an equal volume of ADP-Glo Reagent was added to terminate the kinase reaction, and the resultant ADP level was measured according to the manufacturer's instructions. The light generated by the luciferase/luciferin reaction was measured using the TriStar LB941 Luminometer.

\subsection{Computational system for MD simulation}

The initial structural data of the human ALK complexed with crizotinib, alectinib, ceritinib, lorlatinib, and brigatinib were obtained from the Protein Data Bank (PDB code: 2XP2, 3AOX, 4MKC, 4CLI, and $5 \mathrm{~J} 7 \mathrm{H}$, respectively). The structures of disordered loops and flexible side chains were modeled using the structure preparation module in the Molecular Operating Environment (MOE, Chemical Computing Group, Montreal, Canada; version 2013.08) [16]. The N- and Ctermini of the protein models were capped with acetyl and $\mathrm{N}$-methyl groups, respectively. The dominant protonation state at $\mathrm{pH} 7.0$ was assigned for titratable residues. Each mutation was introduced into the structures of wild-type ALK using the structure preparation module in MOE.

Crizotinib, alectinib, ceritinib, lorlatinib, and brigatinib were protonated to form ionization states in the solution, and their net charges were set to $+1,+1,+1,0$, and +2 , respectively. Each structure of the ALKTKIs was optimized, and the electrostatic potential was calculated at the HF/6-31G* level using the GAMESS program [17], after which the atomic partial charges were obtained by the RESP approach [18]. The other parameters for the compounds were determined by the general Amber force field (GAFF) [19] using the antechamber module of AMBER Tools 12. The Amber ff99 force field was used for protein and ions [20], and TIP3P was used for water molecules [21]. Water molecules were placed around the complex model with an encompassing distance of $8 \AA$, including approximately $12,000-13,000$ water molecules. Next, charge-neutralizing ions were added to neutralize the system. 


\subsection{MD simulation and binding free energy estimation}

All MD simulations were performed in periodic boundary conditions using the GROMACS 4 program [22]. We used the same simulation parameters as described by Araki et al. [23]. After each of the fully solvated systems was energy-minimized, it was equilibrated for $100 \mathrm{ps}$ under the constant number of molecules, volume, and temperature conditions (NVT), and run for 100 ps under constant numbers of molecules, pressure, and temperature condition (NPT), with positional restraints on the protein's heavy atoms and compound atoms. The production runs were conducted under the NPT condition without the positional restraints. Five sets of 50 ns production runs and three sets of 20 ns production runs were performed with different velocities for the individual ALK-compound complex system and solvated compound system, respectively. The ALK-compound binding free energy $(\Delta G)$ was calculated by MP-CAFEE (Massively Parallel Computation of Absolute binding Free Energy with well-Equilibrated states), which is one of the alchemical free energy perturbation methods, especially the double annihilation method [24]. $\Delta \mathrm{G}$ for each combination of ALK mutants and ALK-TKIs was computed according to a protocol described in our previous study [23]. All free energy simulations were performed on the K-computer (RIKEN, Japan).

\subsection{FMO calculation}

To analyze the interaction of alectinib-L1256 (ALK-I1171N) and alectinib-F1256 (ALK-I1171N + L1256F), Fragment Molecular Orbital (FMO) calculations were performed as follows. We extracted 5000 structures of the ALK-alectinib complex from $50 \mathrm{~ns} \times 5 \mathrm{MD}$ trajectories every 50 ps. 22 residues located within 5 Å from the bound-alectinib were defined as forming the drug-binding pocket. After the backbone $\mathrm{C} \alpha$ atoms were structurally aligned, tertiary structures of these residues and alectinib in the 5000 snapshots were clustered into 10 categories by using the k-means clustering method. For each clustering category, the structure that has the smallest r.m.s. deviation from the cluster center was selected as a representative structure. A total of 10 representative structures of the ALK-alectinib complex were extracted for each of ALK-I1171N and I1171N + L1256F mutants, and further analyzed by FMO calculations. In FMO calculations, amino acid residues and alectinib molecule were treated as a single fragment. Energy calculations was performed at RHF and MP2 levels of theory with resolution of the identity (RI) approximation [25] using cc-pVDZ basis set [26]. The interaction energies of alectinib-L1256 and -F1256 were evaluated from the weighted averaged inter fragment interaction energy (IFIE) over the 10 representative structures. In this study, PAICS program [27] was used for the FMO calculations.

\subsection{Drug screening}

Drug screening was performed as previously described using the SCADS Inhibitor Kit-1, 3 or 4 provided by the Screening Committee of Anticancer Drugs supported by a Grant-in-Aid for Scientific Research on Innovative Areas, Scientific Support Programs for Cancer Research, from the Ministry of Education, Culture, Sports, and Technology of Japan [28]. Parental Ba/F3 cells, EML4-ALK variant 1 wild-type or EML4-ALK-G1202R + L1196M expressing Ba/F3 cells, were treated with the indicated concentration of inhibitors for $72 \mathrm{~h}$.

\section{Results}

\subsection{Resistance mechanisms in alectinib refractory patients}

We obtained biopsy specimens and malignant pleural fluid samples from three relapsed patients following treatment with alectinib (JFCR041 and JFCR-009 were treated with alectinib as the first-line therapy, and JFCR-043 was treated as the second-line therapy after relapse on crizotinib). By sequencing the resistant tumor cells, we also found a G1202R mutation and I1171N mutations (Fig. 1a, b and c). We established cell lines with an I1171N mutation (JFCR-043) from inguinal lymph node biopsy and a G1202R mutation (JFCR041-2) from the pleural effusion. Then, we checked the ALK-TKIs sensitivities of these patient-derived JFCR-043 or JFCR-041-2 cells. As expected from the literature, the JFCR-043 cells harboring an I1171N mutation were sensitive to ceritinib or lorlatinib, but the JFCR-041-2 cells harboring a G1202R mutation were only sensitive to lorlatinib in our in vitro and in vivo studies (Fig. 1d, e, f and Supplementary Fig. 1a-d). Indeed, as shown in Supplementary Fig. 1e, one patient with an I1171N mutation had received ceritinib after alectinib, and showed partial response. However, the tumor eventually relapsed with a G1202R mutation. According to the medical history, after the ceritinib treatment, the patient received chemotherapy, and the recurrent tumor was shown to contain G1202R-mutation harboring cancer cells as the dominant population, but the $11171 \mathrm{~N}$-harboring cancer cells still existed as a minor population in the pleural effusion (no G1202R mutation was detectable by deep sequencing before the ceritinib treatment).

3.2. Identification of a lorlatinib-resistant G1202R + G1269A double mutation in a mouse xenograft model using patient-derived ALK-G1202R positive cells

To investigate the lorlatinib resistance mechanisms on G1202Rmutated ALK-positive NSCLCs, we subcutaneously transplanted patient-derived JFCR-041-2 cells (Fig. 1e, EML4-ALK variant3-G1202R) into the BALB-c nu/nu mice and treated them with increasing concentrations of lorlatinib. Initially, the tumor dramatically shrunk with the lorlatinib treatment, but it eventually started to re-grow, and we used the established lorlatinib-resistant JFCR-041-2 tumors (JFCR-041-2 LorR) for our experiments (Fig. 2a). We discovered an ALK-G1202R + G1269A double mutation by sequencing of the ALK kinase domain from the JFCR-041-2 LorR cells (Fig. 2b). ALK-G1202R + G1269A has been reported as a lorlatinib-resistant mutant, but an alternative drug has yet tobe identified [12]. We treated the JFCR-041-2 LorR cells harboring G1202R + G1269A with crizotinib, alectinib, ceritinib, brigatinib, or lorlatinib, and we analyzed the phosphorylation of ALK and its downstream signals by immunoblotting to confirm whether ALK-G1202R + G1269A double mutants were resistant to lorlatinib and checked the sensitivity of the mutants to the other ALK-TKIs. The ALK autophosphorylation and the phosphorylation of ALK downstream signaling molecules in the JFCR-041-2 LorR cells were maintained under crizotinib, alectinib, and ceritinib but were slightly decreased by lorlatinib and brigatinib. However, the autophosphorylation of ALK was more attenuated under treatment with brigatinib than with lorlatinib (Fig. 2c). These results suggest that the ALK-G1202R + G1269A double mutation confers resistance to lorlatinib, but the mutants remain sensitive to brigatinib. To check this hypothesis, we performed in vivo experiments using the BALB-c nu/nu mouse model bearing JFCR-041-2 or JFCR-0412-LorR. As a result, the growth of JFCR-041-2 LorR cells was inhibited by the treatment with brigatinib $(50 \mathrm{mg} / \mathrm{kg}$ ) compared with the treatment with vehicle control, with alectinib- $(50 \mathrm{mg} / \mathrm{kg}$ ) or lorlatinib$(10 \mathrm{mg} / \mathrm{kg}$ ) treated groups (Supplementary Fig. 2a-d). Because several groups demonstrated that plasma levels of brigatinib at $50 \mathrm{mg} / \mathrm{kg}$ in mice were similar to $180 \mathrm{mg}$ qd in patients, we determined the dose for mice at $50 \mathrm{mg} / \mathrm{kg}[29,30]$. Moreover, we introduced the ALK$\mathrm{G} 1202 \mathrm{R}+\mathrm{G} 1269 \mathrm{~A}$ mutation to the Ba/F3 cells, assessed their cell viability, and performed immunoblotting experiments. We found that the Ba/F3-ALK-G1202R + G1269A cells were resistant to lorlatinib but moderately sensitive to brigatinib, similar to the ALK-G1202R single mutant (Fig. 2c and d and Supplementary Table A). The results of these in vivo and in vitro studies suggest that brigatinib may show some effect on lorlatinib-resistant cells harboring ALK-G1202R + G1269A double mutations. However, G1202R was also found from 
a

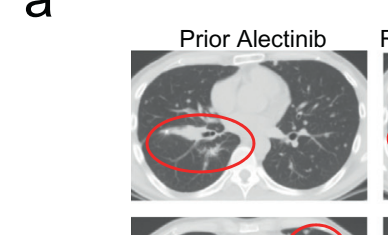

Response to Alectinib

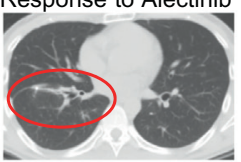

JFCR-041
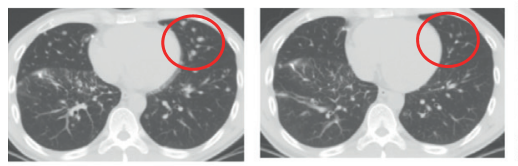

Prior Ceritinib
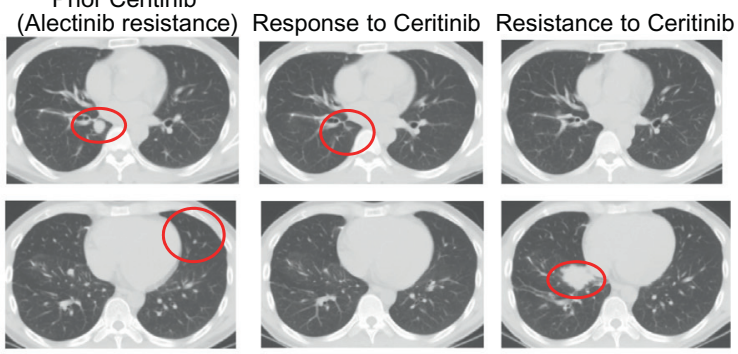

Resistance

mutation

G1202R
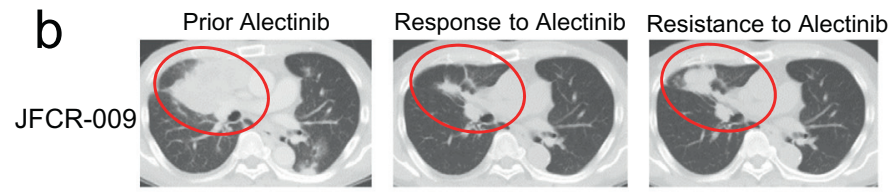

Resistance mutation

$11171 \mathrm{~N}$

I1171N
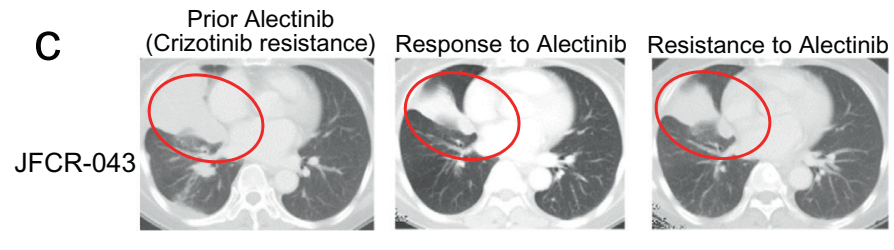

d

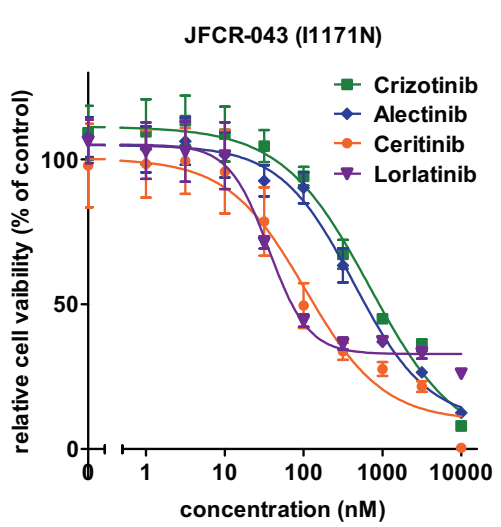

e

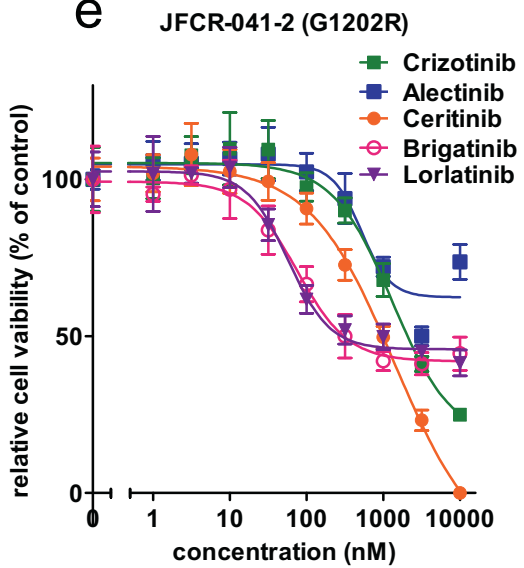

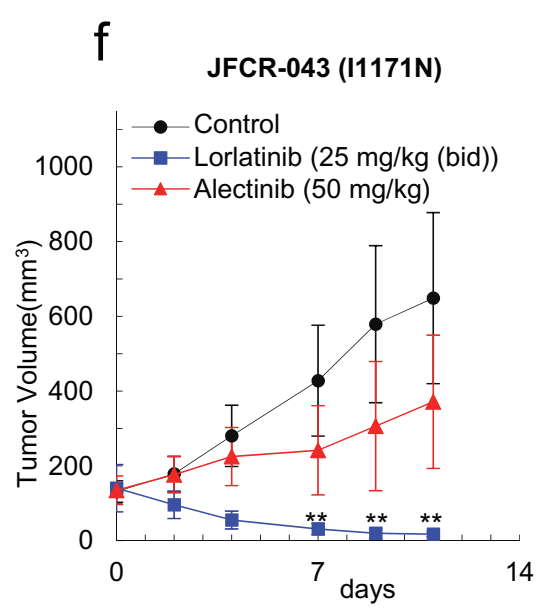

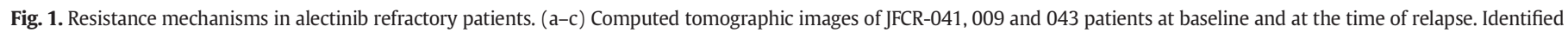

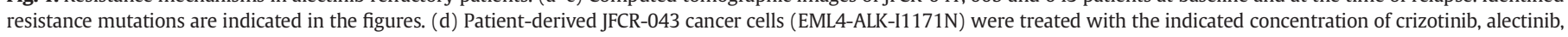

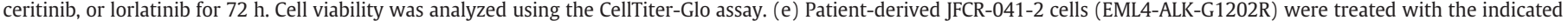

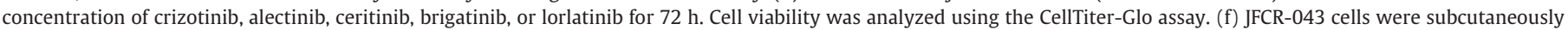

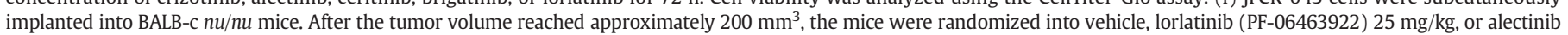
$50 \mathrm{mg} / \mathrm{kg}$ (each group $n=6$ ) and treated once daily by oral gavage for 5 to 6 days/week. The tumor size was measured twice weekly. Results are expressed as mean \pm SD.

several relapse patients following the use of brigatinib. Therefore, this double mutation may not be overcome by brigatinib in the patients.

\subsection{Identification of the crizotinib- or alectinib-resistant ALK mutations from ENU mutagenesis screening}

To validate ENU mutagenesis screening methodology at first, we explored crizotinib or alectinib-resistant ALK mutants using $\mathrm{Ba} / \mathrm{F} 3$ cells expressing EML4-ALK-wild-type clonal cells\#13. As the results, C1156Y, I1171T, F1174C/I/V, L1196M, and I1268L mutations in ALK were identified as crizotinib-resistant mutants. In addition, I1171N/S/T, F1174I/V, and L1196M/Q mutations in ALK were obtained from alectinib-resistant cells (Supplementary Fig. 3a-c). Among these uncovered resistant mutations, we found that the L1196Q mutant, with a relatively uncharacterized alectinib-resistant mutation, was sensitive to ceritinib but resistant to crizotinib; and the crizotinib-

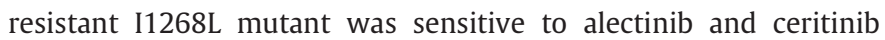
(Supplementary Fig 3a, c, d and e). In addition, we identified a new alectinib-resistant compound mutation V1185L + L1196M (Supplementary Fig. 3a). To confirm the resistance of this V1185L + L1196M double mutation, we generated Ba/F3 cells expressing ALK- V1185L, - L1196M, or - V1185L + L1196M mutations. The cells with a V1185L mutation by itself did not show any resistance to alectinib, however, those with V1185L + L1196M mutations showed a higher resistance to alectinib than the cells with the L1196M mutation alone. Of note, these V1185L, L1196M, and V1185L + L1196M mutants were all similarly sensitive to ceritinib (Supplementary Fig. 3d and f). 
a

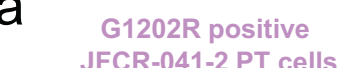

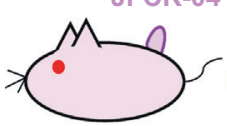

(BALB-C nu/nu mice)

\section{Lorlatinib} $10 \mathrm{mg} / \mathrm{kg}$

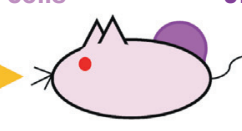

Re-transplantation

$30 \mathrm{mg} / \mathrm{kg}$
G1202R positive JFCR-041-2 LorR cells

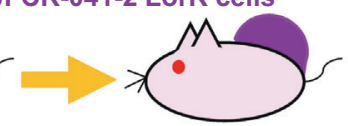

$50 \mathrm{mg} / \mathrm{kg}$ b

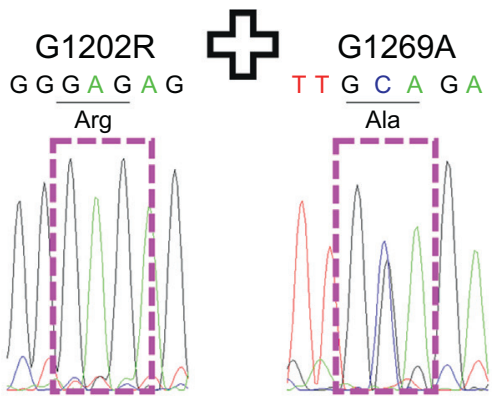

C

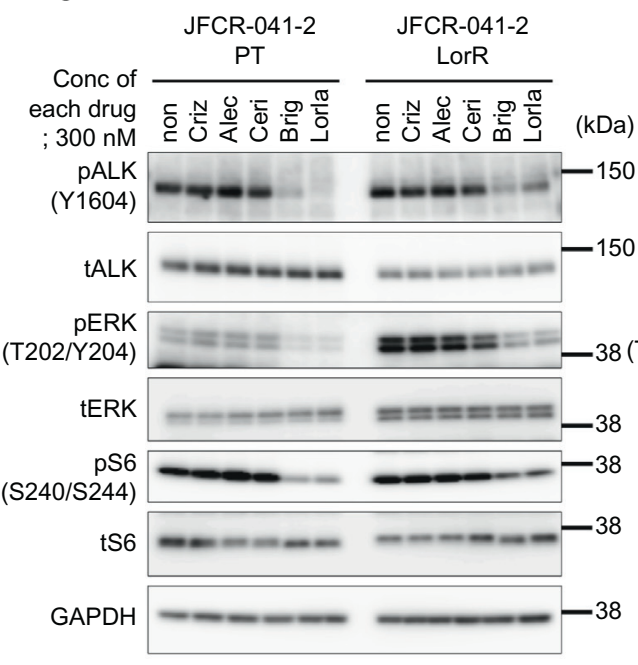

d
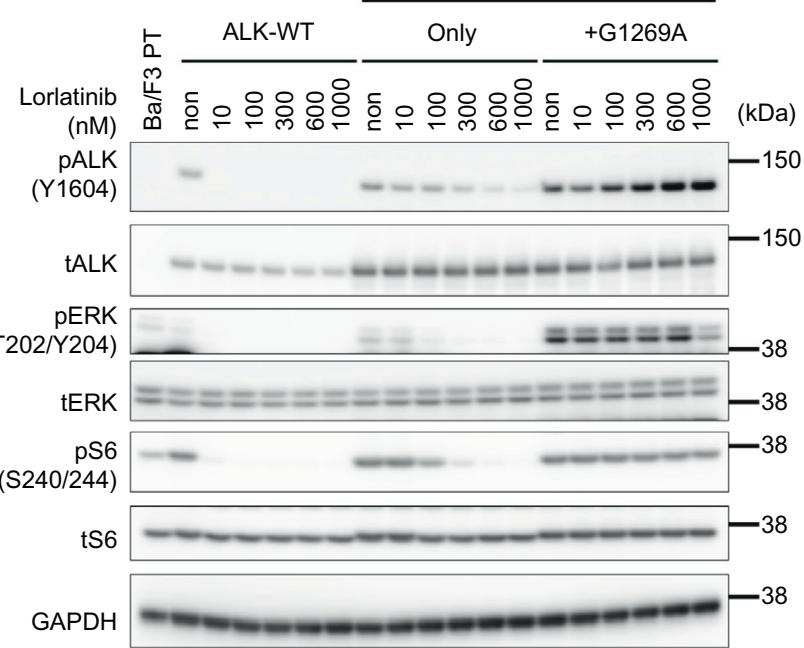

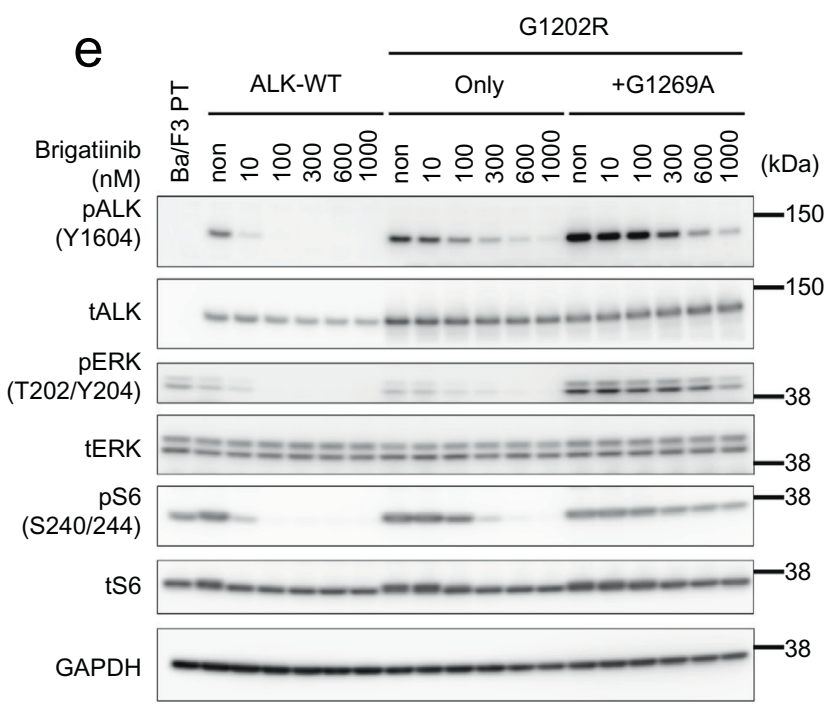

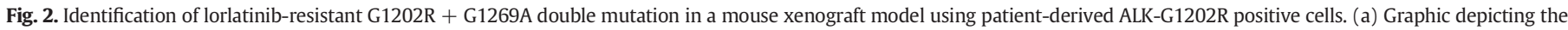

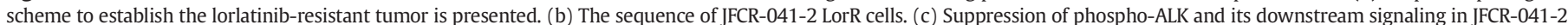

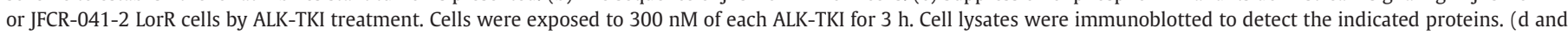

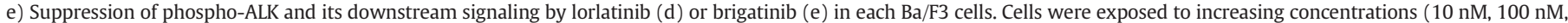
$300 \mathrm{nM}, 600 \mathrm{nM}$ and $1000 \mathrm{nM}$ ) of each inhibitor for $3 \mathrm{~h}$. Cell lysates were immunoblotted to detect the indicated proteins.

3.4. Discovery of the lorlatinib-resistant ALK-I1171N or -G1202R compound mutations, and the therapeutic strategies to overcome them

Since we could discover the previously reported crizotinib- or alectinib-resistant ALK mutants from our ENU mutagenesis screening, we performed ENU mutagenesis screening to identify ceritinib- or lorlatinib-resistant ALK-compound mutation. From the EML4-ALKI1171N harboring Ba/F3 cells, we discovered I1171N + L1198F, + L1196M, +T1151K, + C1156Y, + F1174I, + F1174L, + L1198H, + L1256F, and + G1269A double mutations in the ALK kinase domain 
by sequencing analysis. In addition, we identified several G1202R double mutations: G1202R + L1198F, + L1196M, + F1174C, or + F1174L. In total, we discovered 13 ALK I1171N or G1202R compound mutants (Fig. 3a-d, Supplementary Fig. 4a). ALK-I1171N and ALKG1202R compound mutants showed 2.7-74- and 1.5-35-fold higher
$\mathrm{IC}_{50}$ to lorlatinib than each single mutation, respectively (Fig. 3e, $\mathrm{f}$ and Supplementary Tables B-F). ALK-I1171N + L1198F, I1171N + C1156Y, G1202R + L1196M, and G1202R + F1174L were already identified in clinic as ceritinib- or lorlatinib-resistant ALK mutations [9]. However, because the therapeutic strategies to these ALK-

b

a Lorlatinib-resistant

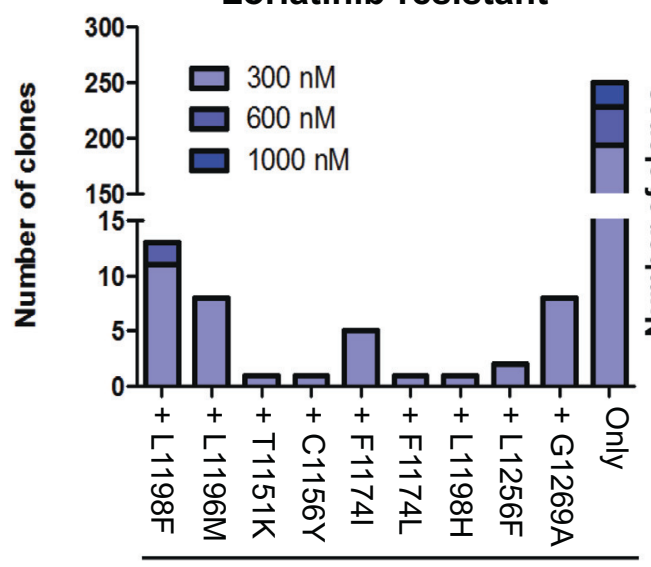

I1171N

C

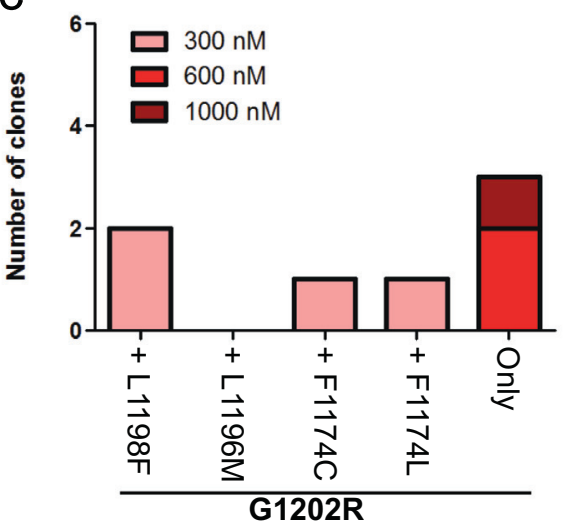

e

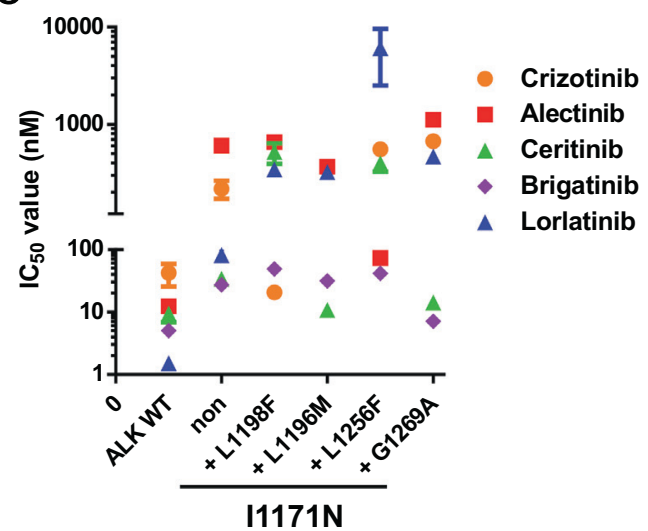

Ceritinib-resistant

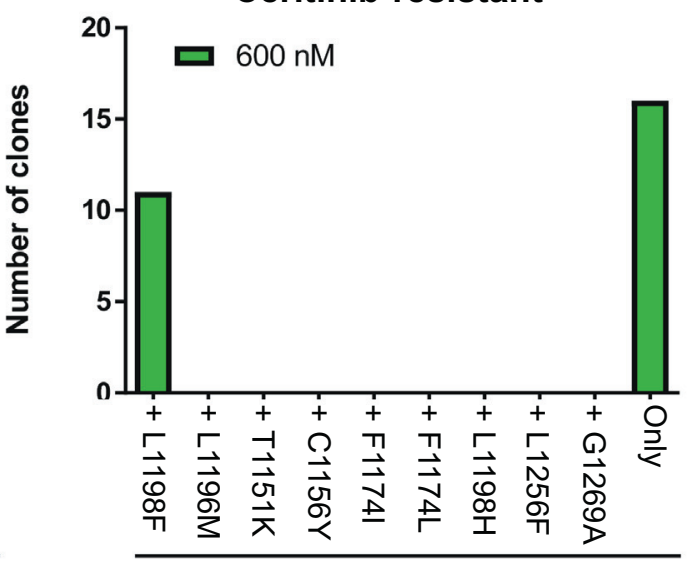

I1171N

d

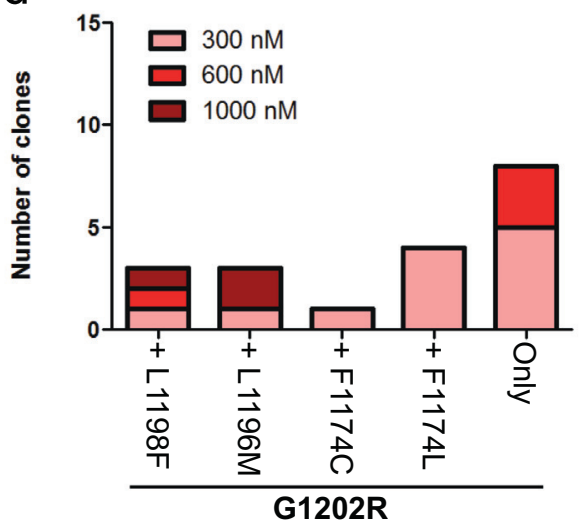

f

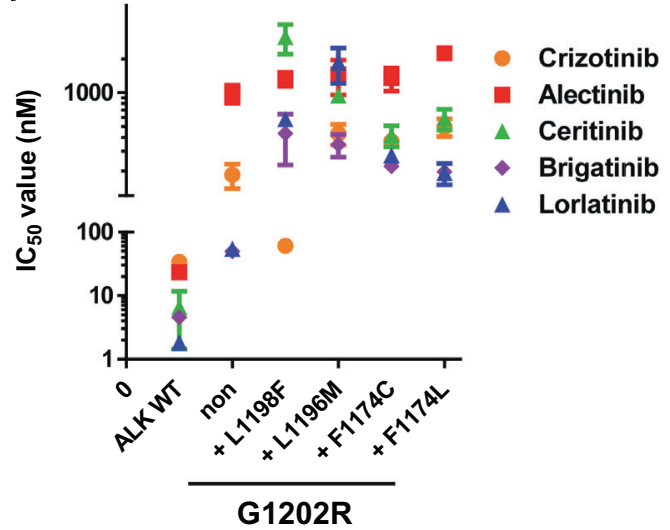

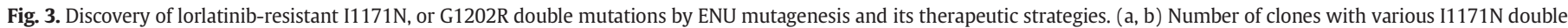

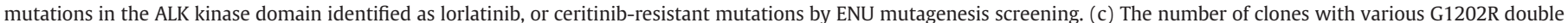

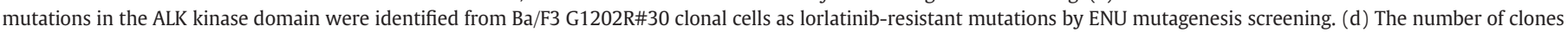

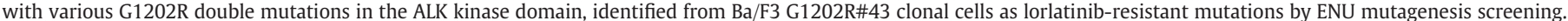

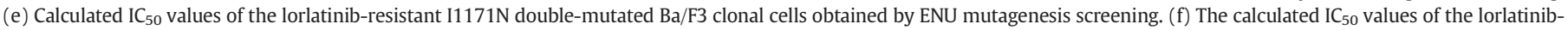

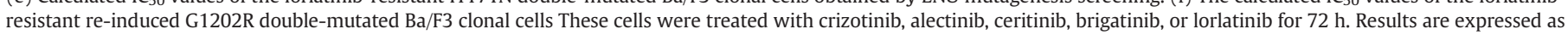
mean \pm SD. 

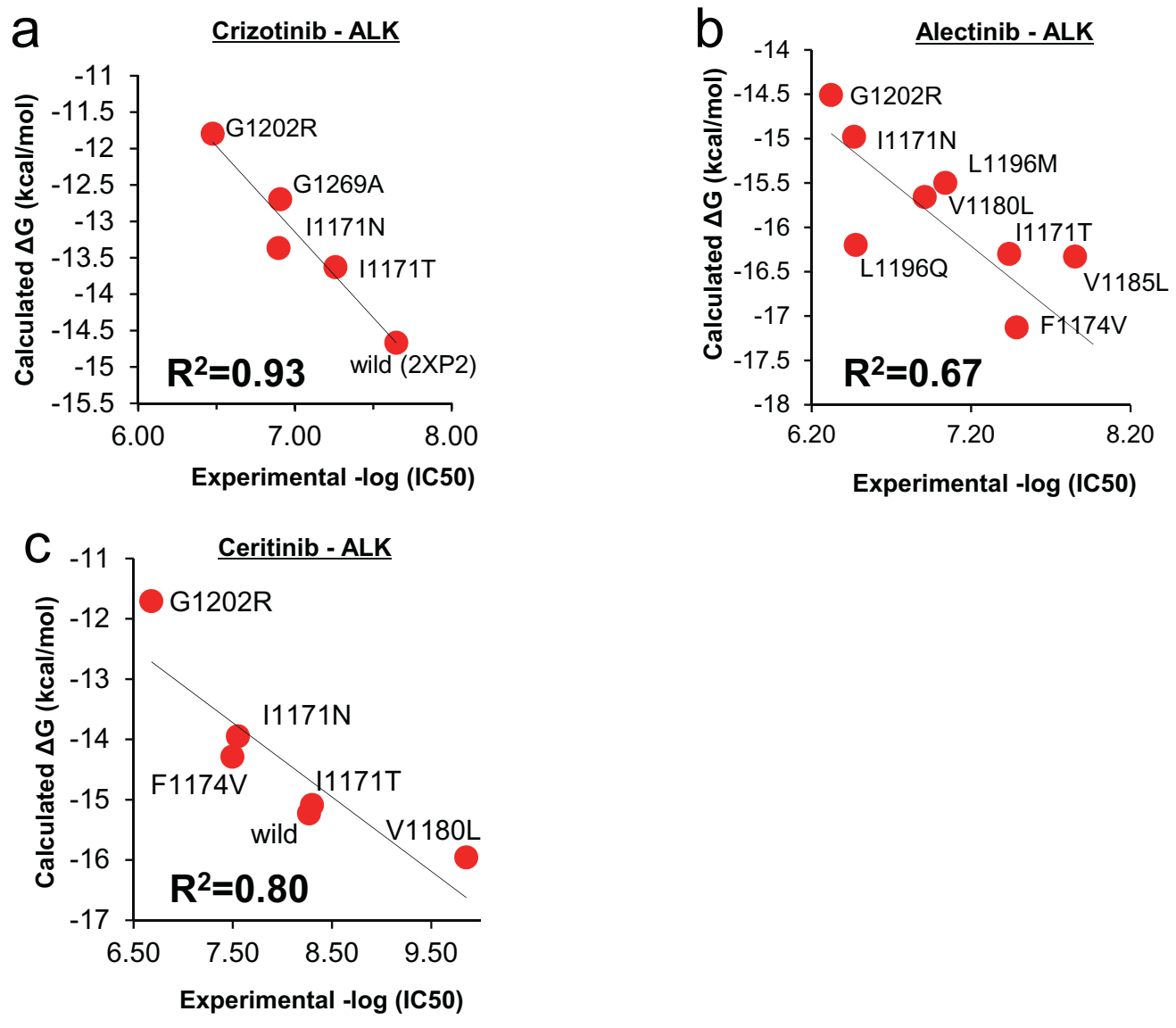

d

Estimated interaction energies of the complexes

\begin{tabular}{lcrrr}
\hline & $\Delta \mathrm{G}$ & coulomb & vdw \\
\hline ALK (wild)-Ceritinib (green) & $-14.86 \pm 1.45$ & $-1.91 \pm 0.63$ & $-12.95 \pm 0.85$ & \\
L1198F-Ceritinib (cyan) & $-13.47 \pm 1.06$ & $-1.81 \pm 0.06$ & $-11.66 \pm 0.07$ & \\
& & & & \\
& & & & \\
ALK (wild)-Crizotinib (green) & $-14.30 \pm 0.49$ & $-1.10 \pm 0.71$ & $-13.20 \pm 1.20$ & \\
L1198F-Crizotinib (cyan) & $-15.14 \pm 0.63$ & $-1.64 \pm 0.11$ & $-13.49 \pm 0.61$ & (kcal/mol) \\
\hline
\end{tabular}

e

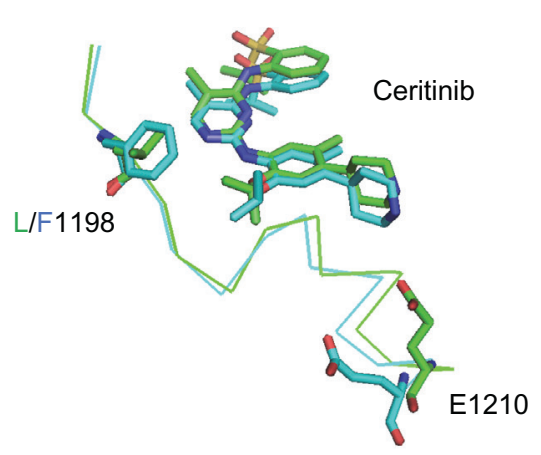

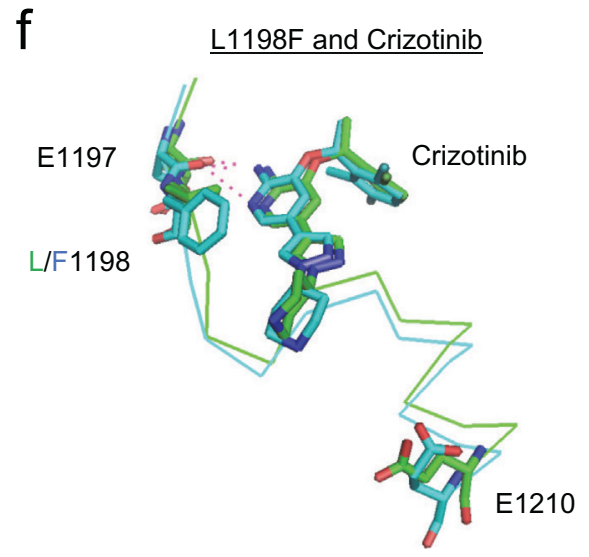


a

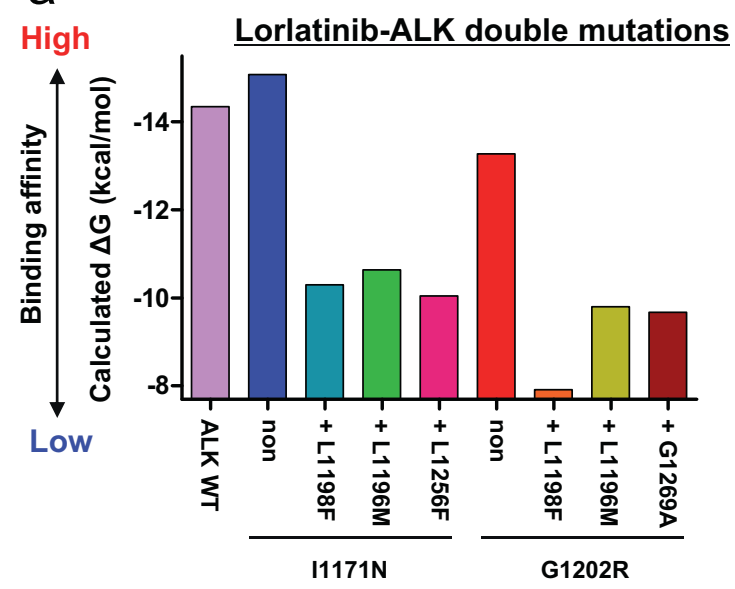

b

\section{High Crizotinib-ALK double mutations}

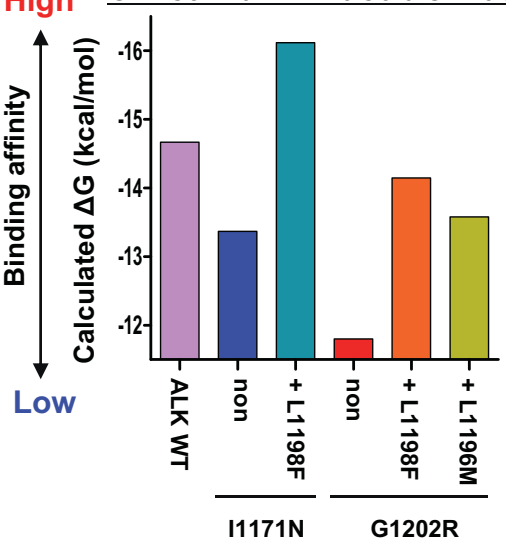

C

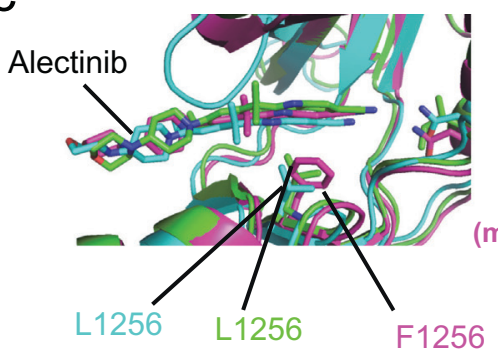

ALK-WT with alectinib

green: $\Delta \mathrm{G}=-\mathbf{- 1 7 . 8 6} \pm 0.62 \mathrm{kcal} / \mathrm{mol}$ )

ALK-I1171 $\mathrm{N}$ with alectinib

(cyan: $\Delta \mathrm{G}=-15.09 \pm 0.75 \mathrm{kcal} / \mathrm{mol}$ )

ALK-I1171 N + L1256F with alectinib

(magenta: $\Delta \mathrm{G}=-15.14 \pm 0.73 \mathrm{kcal} / \mathrm{mol}$ )

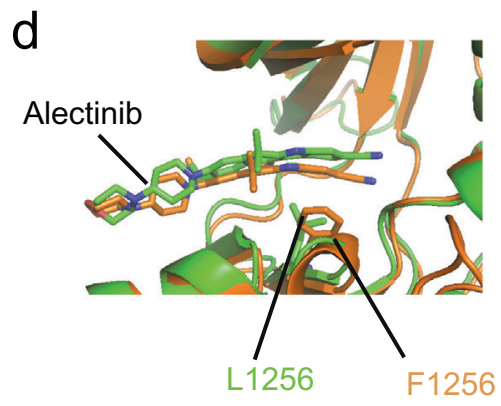

ALK-WT with alectinib

(green: $\Delta G=-17.86 \pm 0.62 \mathrm{kcal} / \mathrm{mol}$ )

ALK-L1256F with alectinib

(orange: $\Delta \mathrm{G}=-16.53 \pm 1.04 \mathrm{kcal} / \mathrm{mol}$ )
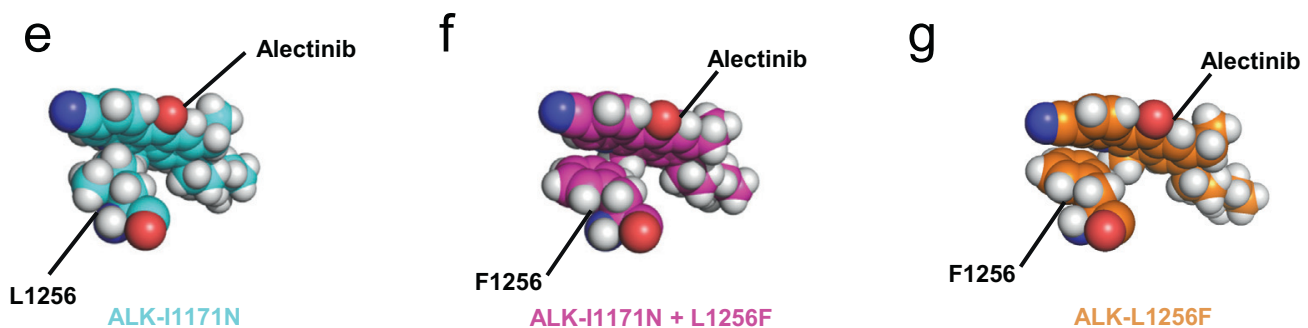

ALK-L1256F

Fig. 5. Computational simulation of the binding affinity between ALK compound mutants and ALK-TKIs by MP-CAFEE and FMO calculations. (a and b) The calculate $\Delta G$ values of lorlatinib (a), or crizotinib (b) to each ALK double mutant are shown in the bar graph. (c, d) MD-relaxed structures of alectinib-bound ALK-WT (green), I1171N (cyan), I1171N + L1256F (magenta), and L1256F single (orange). I/N1171, L/F1256, and alectinib in an energetically stable conformation obtained from five sets of 50 ns simulations are depicted by sticks (green/cyan/ magenta, carbon; blue, nitrogen; red, oxygen). Average $\Delta \mathrm{G}$ values (from three sets of the free energy simulations) are indicated. (e-g) The position of L/F1256 and alectinib in the tertiary structure of (e) ALK-I1171N (cyan), (f) ALK-I1171N + L1256F (magenta), or (g) ALK-L1256F single (orange) mutant.

Fig. 4. Computational prediction of the binding affinity between ALK mutants and ALK-TKIs. (a-c) The binding free energy $(\Delta G)$ of crizotinib (a), alectinib (b), or ceritinib (c) to WT or each resistant mutant is plotted against experimental IC $\mathrm{C}_{50}$ of the corresponding Ba/F3 mutant. These $\Delta \mathrm{G}$ values are calculated by MP-CAFEE, which is one of the MD free energy simulation methods. Solid lines represent linear fits with square of the correlation coefficients $\left(R^{2}\right)$ of $0.93,0.67$, and 0.80 for crizotinib-ALK, alectinib-ALK, and ceritinib-ALK plots, respectively. (d) The average binding free energies ( $\Delta \mathrm{G}$ values) of ceritinib or crizotinib (from three sets of free energy simulations). Electrostatic (coulomb) and van der Waals (vdw) contributions in $\Delta G$ values indicate that this mutation induces a slight increase in electrostatic interactions with crizotinib but decreases the van der Waals contacts with ceritinib. (e and f) Conformational differences in the drug-binding site between ALK-WT (green) and L1198F mutant (cyan). E1197, L/F1198, E1210, and ceritinib (e) or crizotinib (f) in an energetically stable conformation obtained from five sets of 50 ns simulations are depicted by sticks (green/cyan, carbon; blue, nitrogen; red, oxygen; yellow, sulfur). ALK-TKI hydrogen bonds are depicted by dashed lines. 
a

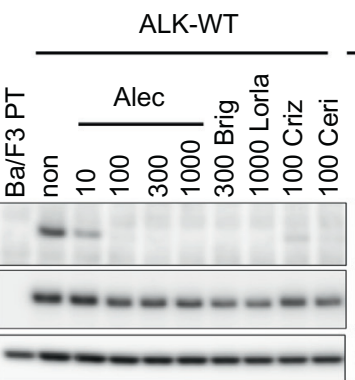

ALK-I1171N

Alec

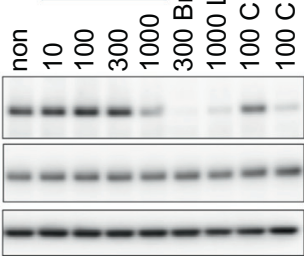

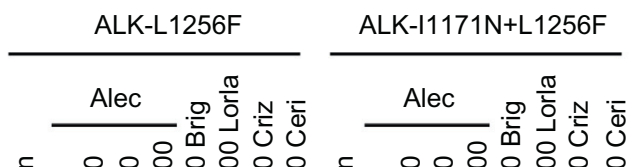

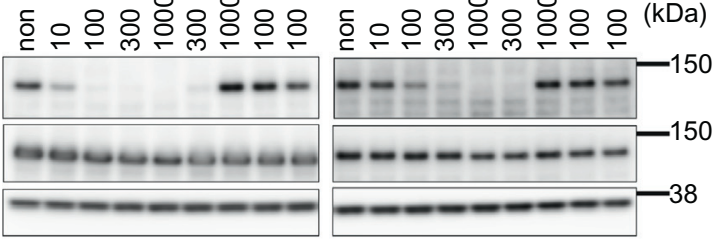

b

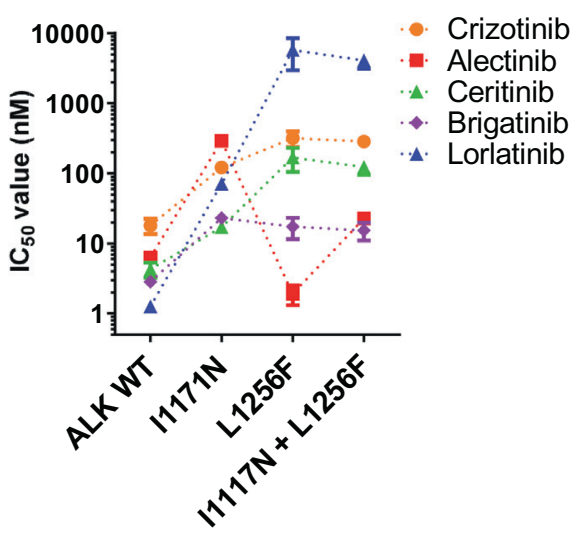

d

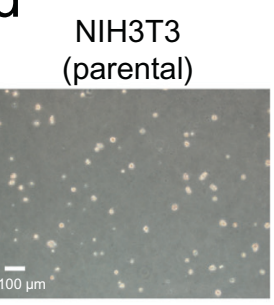

e

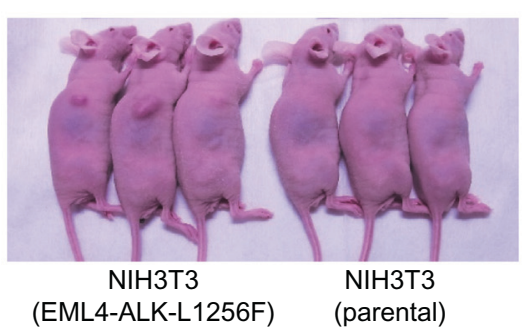

$f$

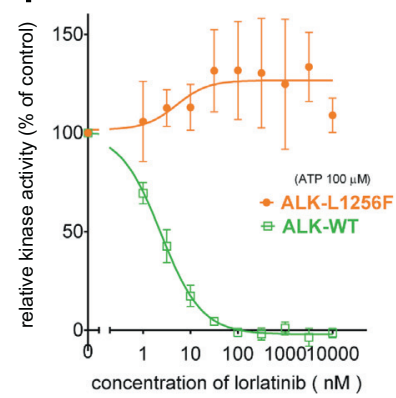

NIH3T3 (EML4-ALK-WT)

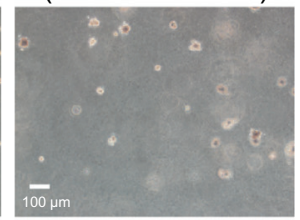

C

Residue of ALK-WT

Residue of ALK-L1256F

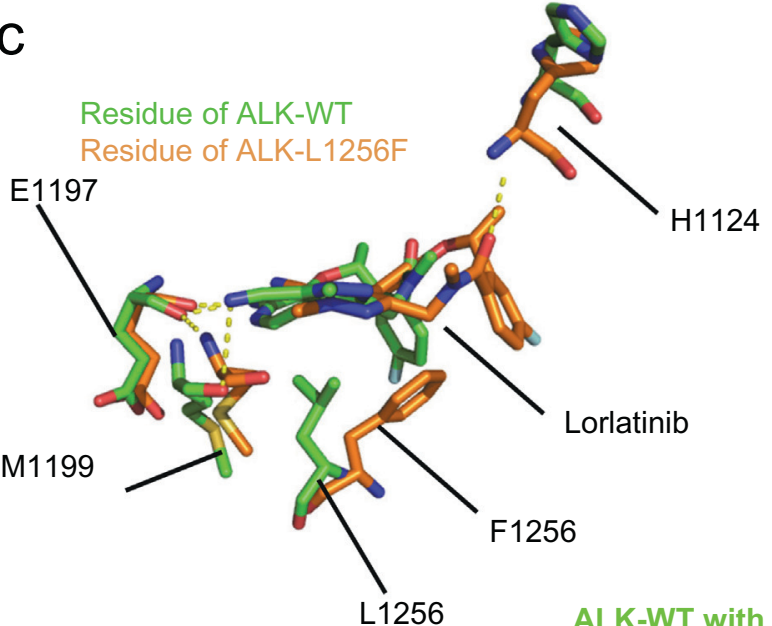

M1199

NIH3T3

(EML4-ALK-L1256F)

(green: $\Delta \mathrm{G}=--14.34 \pm 1.33 \mathrm{kcal} / \mathrm{mol}$ )

ALK-L1256F with lorlatinib (orange: $\Delta \mathrm{G}=-10.05 \pm 1.78 \mathrm{kcal} / \mathrm{mol}$ )
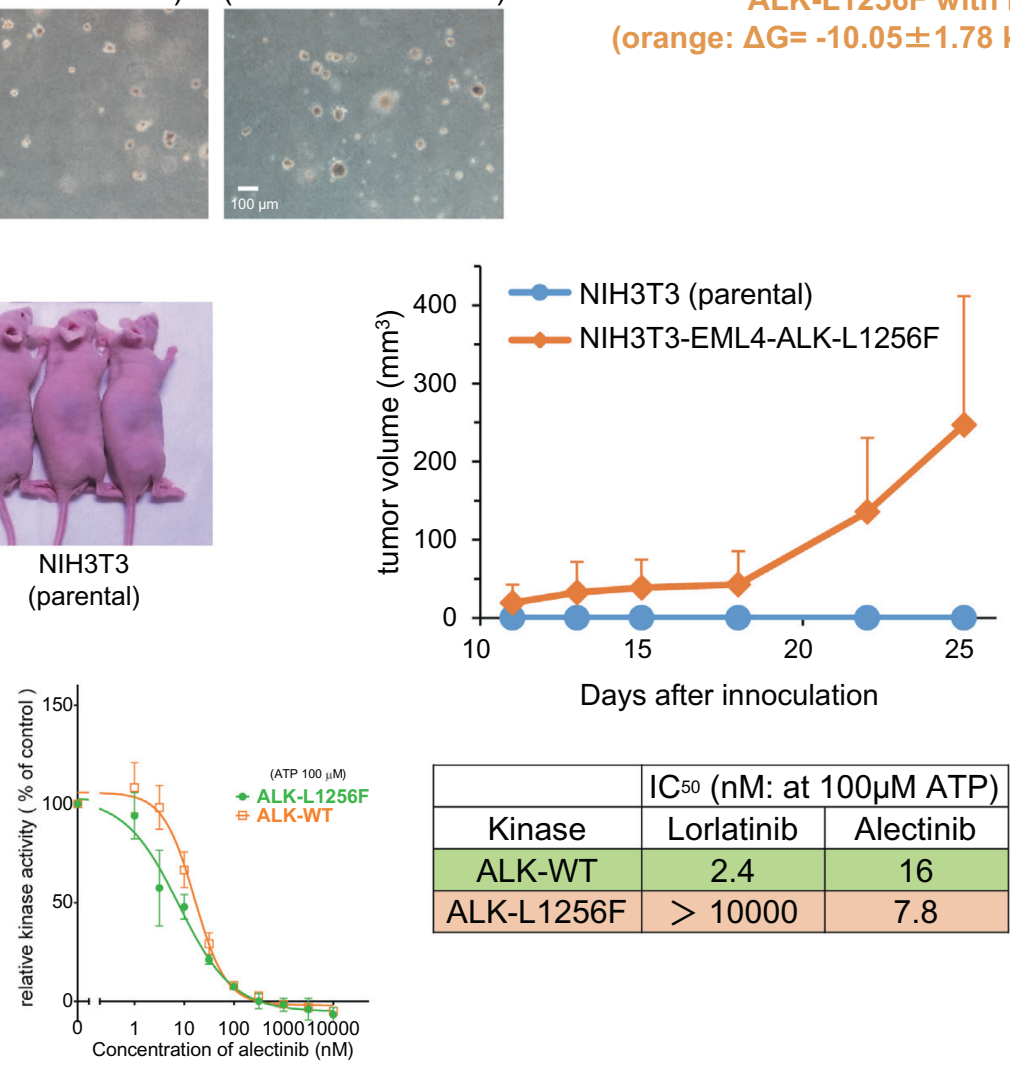

\begin{tabular}{|c|c|c|}
\hline & \multicolumn{2}{|c|}{$\mathrm{IC}_{50}(\mathrm{nM}:$ at $100 \mu \mathrm{M}$ ATP) } \\
\hline Kinase & Lorlatinib & Alectinib \\
\hline ALK-WT & 2.4 & 16 \\
\hline ALK-L1256F & $>10000$ & 7.8 \\
\hline
\end{tabular}


compound mutants have not been clarified yet, we examined the sensitivity of these compound mutants to clinically approved ALK-TKIs such as crizotinib, alectinib, ceritinib, or brigatinib. The early-generation ALKTKIs were found to be effective against some of the ALK-compound mutants, e.g., crizotinib suppressed ALK-G1202R + L1198F and -I1171N $+\mathrm{L} 1198 \mathrm{~F}$ as shown in the previous studies. In addition, alectinib could overcome ALK-I1171N + L1256F, whereas ALK-I1171N single mutant showed high resistance. Furthermore, ceritinib could suppress ALK-I1171N + L1196M and + G1269A, and brigatinib was active against the ALK-I1171N + L1198F, + L1196M, + L1256F, and + G1269A mutants (Fig. 3 and Supplementary Fig. 4b-h, 5 and 6). These results indicate that for certain lorlatinib-resistant compound mutations, an earlier generation ALK-TKI may be able to overcome resistance based on our in vitro studies.

\subsection{Computational approach with MD simulation and FMO calculation for structure analysis of ALK-TKI interactions and binding affinity prediction of resistance mutations}

In our previous study, where we found two alectinib resistance mutations (I1171T and V1180L), we performed computational simulations using MP-CAFEE and succeeded in quantifying the binding affinity of alectinib to these ALK-TKI resistant mutants, and we proposed resistance mechanisms deduced from their MD simulation and the free energy estimation. To strictly assess its prediction accuracy, we applied MP-CAFEE to several ALK mutants resistant to crizotinib, alectinib, or ceritinib, and compared the calculated binding free energy $(\Delta G)$ values with the experimental $\mathrm{IC}_{50}$ values measured from in vitro viability assays using $\mathrm{Ba} / \mathrm{F} 3$ cells expressing these ALK mutants. In addition, to evaluate the relative binding affinity between ALK-TKIs and ALK kinase, we also calculated $\Delta G$ between each ALK mutant with ATP. For all of the three TKIs, we obtained a clear linear correlation between the experimental $\mathrm{IC}_{50}$ and $\Delta \mathrm{G}$ of each TKI relative to that of ATP (Fig. 4a-c). This result suggests that the free energy estimation using MP-CAFEE can correctly predict how each ALK-TKI resistant mutation will affect the drug-binding. Indeed, the L1198F mutation in ALK, which we identified from the analysis of ceritinibresistant cells that were established from H3122 cells continuously passage into media containing higher ceritinib concentrations, are highly sensitive to crizotinib. Our MD simulation reproduced the increased sensitivity for crizotinib and the decreased sensitivity for ceritinib (Fig. 4d-f). Moreover, we applied this free energy estimation method to pairs of lorlatinib, crizotinib, or ceritinib and each ALK double mutant identified in this study. The ALK double mutant with especially high experimental $\mathrm{IC}_{50}$ value to lorlatinib in the cell viability assays showed a significantly-decreased binding affinity for this TKI than each of the single mutants (Fig. 5a). In contrast, ALK-I1171N + L1198F, or ALK-G1202R + L1198F exhibited higher binding affinities for crizotinib than each single mutant (I1171N or G1202R), consistent with the $\mathrm{IC}_{50}$ values obtained by in vitro experiments. As a whole, we confirmed similar trends between $\Delta \mathrm{G}$ values predicted for ALK double mutants and their experimental $\mathrm{IC}_{50}$ values, however, the prediction failed for several mutant-drug pairs (Fig. 5b, and Supplementary Fig. 7a).
3.6. ALK-L1256F single mutation confers resistance to lorlatinib but increases sensitivity to alectinib

To check if ALK-L1256F mutation contributes in alectinib resensitization of ALK-I1171N + L1256F, we tried to study the alectinib sensitivity of ALK-L1256F single or double mutation by using MPCAFEE, and MD simulation methods. However, our MD simulation suggested that ALK-alectinib binding was not significantly affected by the Leu to Phe substitution on residue1256 although this residue is located very near the drug. Therefore, we hypothesized that MP-CAFEE, which is a free energy computation method based on the classical MD simulation, cannot accurately calculate the contribution of the interaction between alectinib and the phenylalanine residue at ALK-1256 through the delocalized $\pi$ electron (Fig. 5c-g). Next, we performed an FMO analysis, which is a quantum chemical method. From the FMO analysis, we can analyze the molecular interaction in large molecules such as proteins by dividing them into small fragments. The FMO-RHF method can calculate the polar interaction energy (electrostatic or charge transfer interaction), and the FMO-MP2 method can assess the non-polar interaction energy (van der Waals or dispersion interaction). Thus, the FMO-RHF and -MP2 calculations revealed reductions not only in the polar interaction but also in the non-polar interaction (the $\pi-\pi$ interactions) between alectinib and ALK-I1171N + L1256F, or ALK-L1256F (Supplementary Fig. 7b and c). It is conceivable that the re-sensitivity of the ALK-I1171N + L1256F mutant to alectinib was the result of increasing the $\pi-\pi$ interactions between the benzocarbazole moiety in alectinib and the phenyl group at ALK-1256.

Next, to examine whether L1256F mutation confer resistance to lorlatinib, we induced EML4-ALK-I1171N + L1256F, EML4-ALKL1256F, or EML4-ALK-WT into H3122 and EML4-ALK-positive lung cancer cells and examined the sensitivity to lorlatinib and alectinib. Both I1171N + L1256F compound mutant and L1256F single mutant cells were found to be resistant to lorlatinib and sensitive to alectinib under in vitro and in vivo conditions. Notably, the I1171N + L1256F compound mutants showed higher resistance to lorlatinib than L1256F alone (Supplementary Fig 8). Because H3122 cells express endogenous EML4-ALK (WT), we next constructed $\mathrm{Ba} / \mathrm{F} 3$ cells expressing ALK-I1171N + L1256F and -L1256F, and confirmed the sensitivity to clinically approved ALK-TKIs. As a result, these two mutations showed resistance to lorlatinib and sensitivity to alectinib than the ALK-WT, and I1171N single mutant (Fig. 6a and b). The MD simulation revealed that F1256 residue clashed with the fluorobenzene group of lorlatinib, resulting in the significant decrease of the van der Waals interaction between lorlatinib and F1256-mutated ALK kinase domain, and that lorlatinib may be extruded from ALK (Fig. 6c). The Ba/F3-EML4-ALK-L1256F cells grew without IL-3, suggesting that ALK-L1256F possessed oncogenicity. To confirm the oncogenicity of L1256F single mutant, we performed soft agar assay and tumor formation assay using NIH3T3 cells expressing EML4-ALK-L1256F. As a result, NIH3T3 cells expressing EML4-ALK-L1256F showed tumorigenicity, although slightly weaker than that of NIH3T3 cells expressing EML4-ALK-WT (Fig. 6d and e). We finally performed in vitro kinase assay to compare the activity of lorlatinib and alectinib against WT- or L1256F-mutated ALK using an ADP-Glo kit. We could confirm that the ALK-L1256F mutant was highly resistant to lorlatinib, but markedly sensitive to alectinib (Fig. 6f).

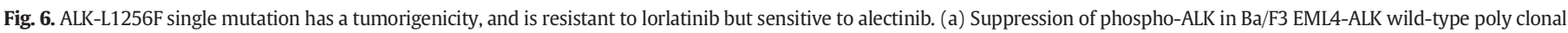

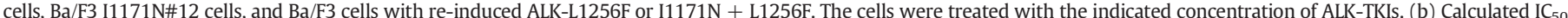

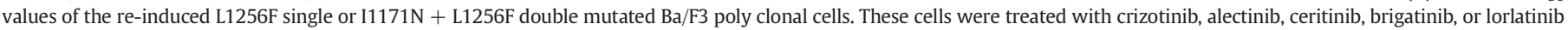

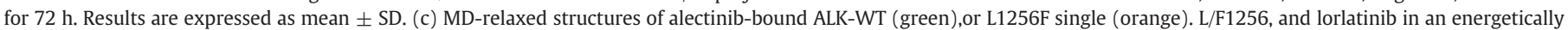

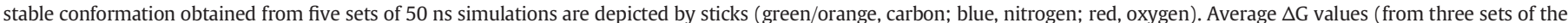

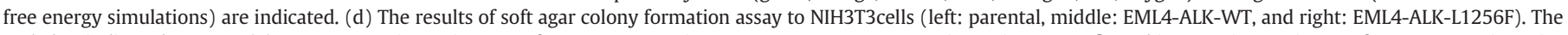

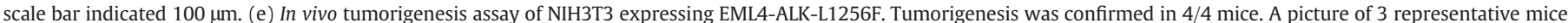

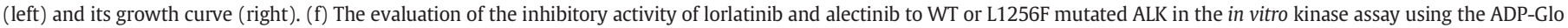
Assay kit. Alectinib, but not lorlatinib, showed a dose-dependent inhibition of ALK-L1256F mutant activity. 

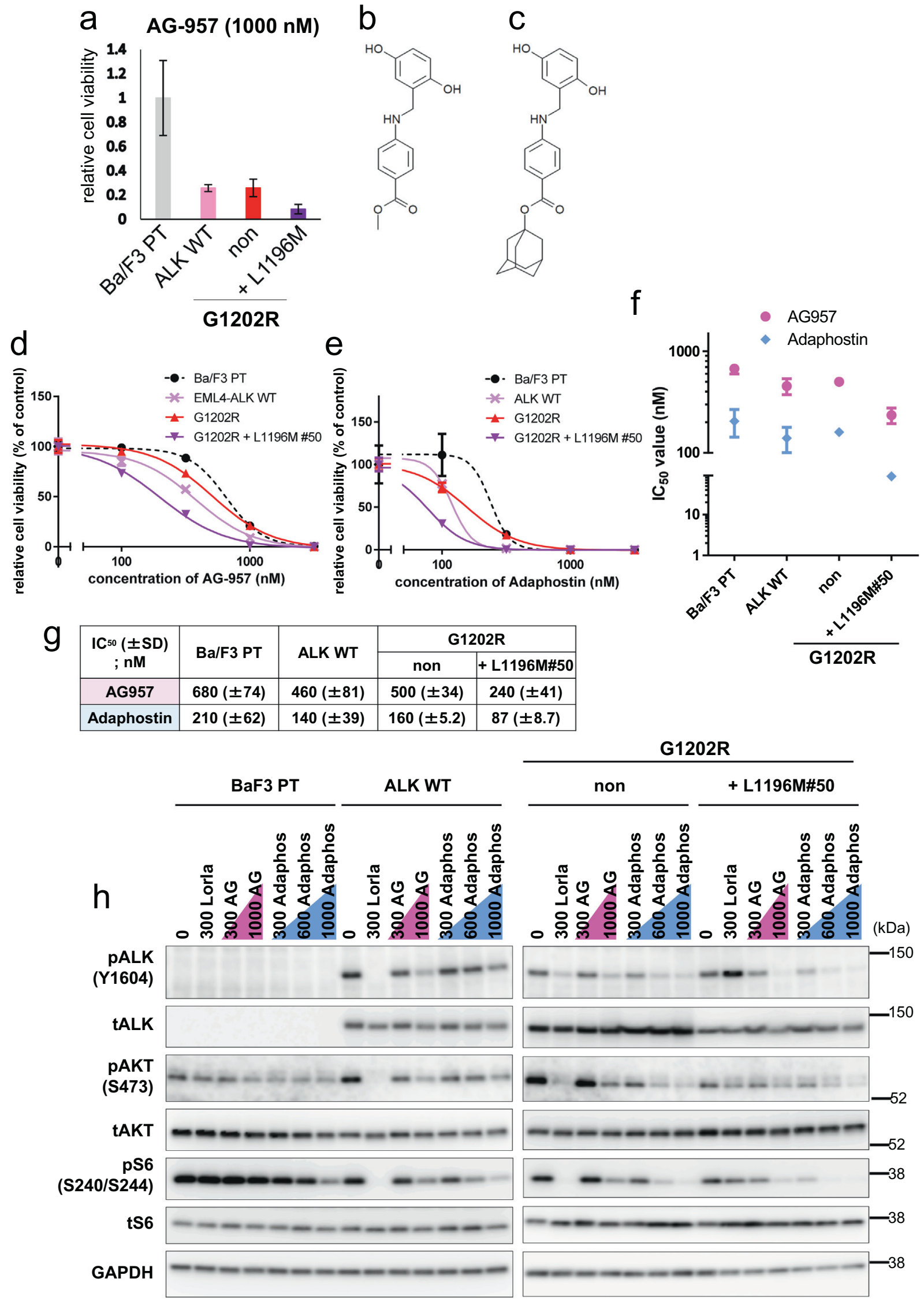

G1202R 


\subsection{Identification of inhibitors of the lorlatinib-resistant ALK-G1202R} + L1196M double mutant

Although ALK-G1202R + L1196M compound mutations, previously reported in clinic and in vitro studies including this study, showed high resistance to all ALK-TKIs, the overcoming drug to this mutant has yet to be discovered. Therefore, we performed high throughput screening with 300 inhibitors with known drug targets. Our results showed AG957, previously developed as a BCR-ABL inhibitor, preferentially suppressed the viability of G1202R + L1196M cells than that of ALK-WT or the ALK-G1202R mutated cells (Fig. 7a and b). Therefore, to investigate the sensitivity to AG-957 in detail, we selected the EML4-ALKG1202R + L1196M expressing BaF3 clonal cells\#50 that showed a similar sensitivity to the HSP90 inhibitor 17-AAG as the Ba/F3 EML4-ALK wild-type, Ba/F3 G1202R, and G1202R + L1196M polyclonal cells. (Supplementary Fig. 9). Previous studies revealed that the stability of EML4-ALK was maintained by HSP90 and the sensitivity to 17-AAG was not changed regardless of the mutations in the ALK kinase domain $[31,32]$. Next, we tested the detailed sensitivity of the G1202R + L1196M\#50 cells to AG-957 and adaphostin, AG-957 analogue (Fig. 7c). G1202R + L1196M cells\#50 showed lower IC 50 values compared to ALK-G1202R, or ALK-WT cells (Fig 7d-g). Because the treatment with AG-957 and adaphostin clearly decreased the autophosphorylation of ALK and the phosphorylation of downstream signals in a dose-dependent manner, it is suggested that AG-957 and adaphostin directly suppressed the ALK-G1202R + L1196M mutations (Fig. 7h).

\section{Discussion}

ALK-I1171N and -G1202R were found to be highly resistant to alectinib in our study as well as in previous reports [9]. Because ALKI1171N or -G1202R conferred resistances are believed to be overcome by ceritinib (only for I1171N) or lorlatinib, treatment with these agents would be useful after the first-line alectinib therapy. When our manuscript was under preparation, Yoda et al. reported various ALKcompound mutations after the second-line lorlatinib treatment [12], but they did not show which drugs would be effective to overcome the resistances. In this study, we also discovered numerous lorlatinibor ceritinib-resistant ALK-compound mutations from both our in vivo mouse model and in vitro ENU mutagenesis screening experiments. The ALK-G1202R + G1269A mutants identified in our in vivo study have already been reported in patients. Further, our ENU mutagenesis screening was able to remarkably recapture several clinically identified ALK-crizotinib-, alectinib-, or lorlatinib-resistant mutations.

In our study, as previously reported in clinically detected lorlatinibresistant ALK-C1156Y + L1198F mutant, we also clearly demonstrated that crizotinib overcame the lorlatinib-resistant ALK-I1171N + L1198F and -G1202R + L1198F mutants. Thus, re-treatment with crizotinib may be effective when additional L1198F mutations are detected. In addition, by computational MD simulation, the present study revealed that the $\Delta G$ value of ALK-L1198F single mutant was lower than that of ALKWT. To our surprise, we discovered the alectinib re-sensitization mutation ALK-I1117N + L1256F. Because ALK-L1256F single mutated cells were extremely sensitive to alectinib, the substitution of Leu with Phe at this position (L1256) rather than a conformational change of ALK kinase domain by $11117 \mathrm{~N}$ and L1256F may be an important factor in alectinib re-sensitization of ALK-I1171N + L1256F (see below for details). To date, the L1256F single mutation has not been reported, and the $\mathrm{L} 1256$ residue is involved in maintaining the structure of the ALK kinase by configuring the $\mathrm{C}$-spine and binding to crizotinib or lorlatinib [33-35]. Importantly, our study and another set of authors reported four ALK-L1256F single or compound mutations as lorlatinibresistance mechanisms [12]. Therefore, various ALK-L1256F single or compound mutations is expected to emerge in relapse patients following the use of lorlatinib in the future. In this study, we proved that ALKL1256F single mutant are highly resistant to lorlatinib and markedly sensitive to alectinib in vitro, in cell lines (Ba/F3 and H3122 cells), and in vivo. In addition, NIH3T3 cells expressing EML4-ALK-L1256F demonstrated tumorigenicity in mouse, although slightly weaker than that exhibited by EML4-ALK-WT. Because I1171N + L1256F mutant showed higher resistance to lorlatinib and higher tumorigenicity than L1256F single mutant, and because lorlatinib is currently approved for the treatment of ALK-TKI treated patients, L1256F compound mutations may emerge in clinical settings in the future. For ALK-L1256F mutation or compound mutations, alectinib (or brigatinib) is expected to overcome the resistance.

Ceritinib was effective against the ALK-I1171N + G1269A and - I1171N + L1196M mutants. However, the cells with the compound G1202R + G1269A or G1202R + L1196M mutations exhibited resistance to it, suggesting that G1202R mutation significantly affects the binding affinity of ceritinib to ALK mutants. Conversely, brigatinib exhibited lower $\mathrm{IC}_{50}$ against four ALK-I1171N compound mutants and demonstrated similar $\mathrm{IC}_{50}$ against both G1202R alone and G1202R + G1269A compound mutation. Brigatinib was shown to be active against G1202R-mutated ALK both under in vitro and in vivo conditions. However, G1202R mutation was observed in approximately 50\% of brigatinib refractory patients' tumor. Thus, in a clinical setting, the efficacy of brigatinib against lorlatinib-resistant G1202R + G1269A mutant may be limited.

Although the ALK-G1202R + L1196M mutants showed high resistance to all clinically approved ALK-TKIs, we found that AG-957 and adaphostin could overcome the resistances. AG-957, which is a member of the tyrphostin family, and the AG-957 analogue adaphostin were developed as BCR-ABL inhibitors. In this study, we found that G1202R + L1196M expressing Ba/F3 showed lower $\mathrm{IC}_{50}$ to AG-957 or adaphostin compared with the BCR-ABL positive K562 cells and did not show any sensitivity to $A B L$ inhibitors, such as imatinib, dasatinib, or ponatinib, thereby differing from K562 cells (Supplementary Fig. 10). Tyrphostin is a compound with a structure similar to that of the tyrosine kinase substrate [36]. AG-957 and adaphostin competitively suppress substrate binding in vitro (adaphostin suppresses both BCR-ABL-wild-type and BCR-ABL-T315I) [37,38]. Considering that AG957 or adaphostin probably bind to the substrate-binding site in the BCR-ABL kinase domain, these agents may also bind to the substratebinding region in the ALK kinase domain, and the substrate-binding site conformation of ALK-G1202R + L1196M may be changed compared with that of the WT or G1202R-mutated ALK. However, estimating the binding mode is currently difficult because the structures of the ALK kinase domain with AG-957 or adaphostin have not been revealed. With the crystal structure analysis of the ALK kinase domain with these inhibitors, it will be possible to develop a potent agent targeting the region other than a conventional drug-binding pocket such as the substrate-binding site.

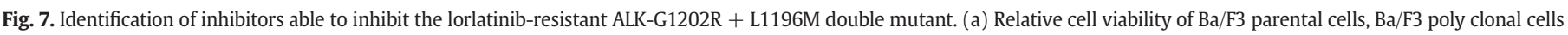

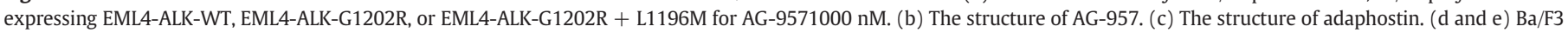

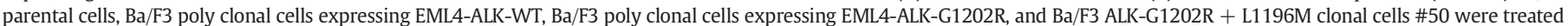

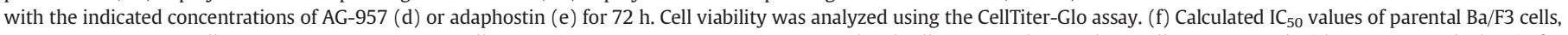

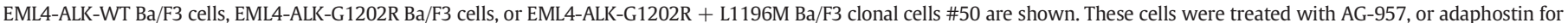

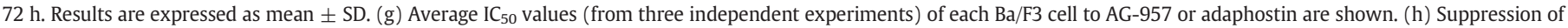

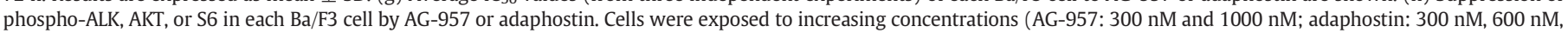
and $1000 \mathrm{nM}$ ) of each inhibitor for $3 \mathrm{~h}$. Cell lysates were immunoblotted to detect the indicated proteins. 
To understand how the mutation affects the ALK-TKI binding affinity, we developed a computational protocol that enable us to calculate the binding free energy $(\Delta G)$ of each ALK-TKI for various ALK mutants and then correlated $\Delta \mathrm{G}$ with the actual experimental $\mathrm{IC}_{50}$. In this study, we found contradicting results between the calculated $\Delta \mathrm{G}$ by MP-CAFEE methods and the cell based study of some mutants. In particular, this method could not detect the difference of $\Delta G$ between alectinib with ALK-I1171N or -I1171N + L1256F. Based on the FMO analysis combined to the MD simulation, we found the possibility that a $\pi-\pi$ interaction between alectinib and the phenyl group in phenylalanine at 1256 in this mutant could cause alectinib re-sensitization by increasing the alectinib-binding affinity, indicating the importance of taking into consideration the quantum chemical calculations that simulate electrical interactions, which cannot be measured by conventional MD simulations alone, for accurate drug resistance prediction in silico. Because the FMO method calculates electrical interactions based on the structural model in a steady state extracted from the MD simulation, it is constrained in that it is greatly influenced by the selection of the steady state to be used in the FMO calculations.

In this study, we chose ENU mutagenesis screening to identify new lorlatinib-resistant mutations, but it has the following two limitations. First, because ENU mutagenesis screening displays a bias for variations of base substitution such as guanine to cytosine [39], it may be difficult to find several mutations such as G1202R with it. For instance, in a G1202R mutation, base substitutions such as G- > A, and G- > C occur and these are less frequently encountered using ENU mutagenesis screenings. Second, because we confirmed only the mutations in the ALK kinase domain, we were not able to detect ALK-TKI resistance mechanisms such as the MET gene amplification or the activating of HER3 [40,41] with our ENU mutagenesis screening. Because other lorlatinib-resistant mechanisms such as small cell transformations are expected, further analysis of lorlatinib resistance mechanisms is important. In addition, because our study did not assume that lorlatinib is treated as a first-line therapy and the therapy of lorlatinib + other ALK-TKIs, we think it would be informative to investigate more detailed analyses of lorlatinib-resistant mechanisms emerging from ALK-TKI naïve $A L K$-rearranged cancer cells. It should be noted that $\mathrm{IC}_{50}$ value of $\mathrm{Ba} / \mathrm{F} 3$ cells showing complete ALK-dependent proliferation is lower than the clinical $\mathrm{IC}_{50}$ value.

In conclusion, our study demonstrates for the first time that some alectinib-lorlatinib-resistant ALK I1171N compound mutants become re-sensitized to clinically approved ALK-TKIs in our preclinical model system. Our study also revealed that ALK L1256F single mutant is resistant to lorlatinib but sensitive to alectinib. Therefore, our study suggests that the clarification of the relapsing mechanisms for patients on lorlatinib through biopsy or liquid-biopsy studies and the search for an effective drug against ALK-compound mutations may become important routine examinations in the near future in clinical settings. In addition, the combination of drug screening and further improvement of in silico simulation might bring an efficiency of drug development in the future.

\section{Funding sources}

This study was supported in part by MEXT/JSPS KAKENHI grant number JP15H02368 and JP17H06327 (to N. Fujita), JP16H04715 (to R. Katayama), JP18K06594 (to M. Araki), the grant from the MHLW/ AMED grant number 18cm0106203h0003 and 18ck0106231h0003 (to R. Katayama), and the grant from the Vehicle Racing Commemorative Foundation (to R. Katayama). MEXT as "Priority Issue on Post-K computer (Building Innovative Drug Discovery Infrastructure Through Functional Control of Biomolecular Systems)" (to Y. Okuno), and FOCUS Establishing Supercomputing Center of Excellence (to Y. Okuno). This research used the computational resources of the $\mathrm{K}$ computer provided by the RIKEN Advanced Institute for Computational Science through the
HPCI System Research Project (Project IDs: hp150272, hp160213, hp170275, hp180186, and ra000018).

\section{Declaration of interests}

Dr. Katayama reports grants from Japan Society for the Promotion of Science (JSPS), grants from Japan Agency for Medical Research and Development (AMED), grants from The Vehicle Racing Commemorative Foundation, during the conduct of the study; grants from TAIHO Pharmaceutical, grants from FUJIFILM, grants from TOPPAN Printing, grants from TAKEDA, personal fees from Pfizer, outside the submitted work; Dr. Araki reports grants from Japan Society for the Promotion of Science (JSPS), grants from the Japanese Ministry of Education, Culture, Sports, Science \& Technology, grants from FOCUS Establishing Supercomputing Center of Excellence, during the conduct of the study;. Dr. Yanagitani reports personal fees from Chugai Pharmaceutical, outside the submitted work;. Dr. Horiike reports grants from MSD K.K., grants from Chugai Pharmaceutical Co., Ltd., grants from Quintiles Transnational Japan K.K., grants from AstraZeneca K.K., grants from Loxo Oncology, Inc., grants from AbbVie GK, personal fees from Chugai Pharmaceutical Co., Ltd., personal fees from AstraZeneca K.K., personal fees from Pfizer Inc., personal fees from ONO PHARMACEUTICAL CO., LTD., personal fees from Boehringer Ingelheim, personal fees from Bristol-Myers Squibb, outside the submitted work;. Dr. Maemondo reports personal fees from Pfizer, personal fees from Chugai, personal fees from Novartis, outside the submitted work;. Dr. Okuno reports grants from the Japanese Ministry of Education, Culture, Sports, Science \& Technology, grants from FOCUS Establishing Supercomputing Center of Excellence, during the conduct of the study;. Dr. Nishio reports grants and personal fees from Ono Pharmaceutical, grants and personal fees from Bristol Myers Squibb, grants and personal fees from Pfizer, grants and personal fees from Chugai Pharmaceutical, grants and personal fees from Eli Lilly, grants and personal fees from Taiho Pharmaceutical, grants and personal fees from AstraZeneca, personal fees from Boehringer-Ingelheim, grants and personal fees from MSD, grants and personal fees from Novartis, personal fees from Sankyo Healthcare, personal fees from Taiho Pharmaceutical, personal fees from Merck Serono, grants from Astellas, outside the submitted work;. Dr. Fujita reports grants from Japan Society for the Promotion of Science (JSPS), grants from Nippon Foundation, during the conduct of the study; grants from Japan Agency for Medical Research and Development (AMED), grants from Api Co., Ltd., outside the submitted work;. Dr. Okada, Dr. Sakashita, Dr. Oh-hara, Dr. Koike, Dr. Watanabe, Dr. Ma, Dr. Tamai, Dr. Kanada, and Dr. Ishikawa have nothing to disclose.

\section{Author contributions}

$\mathrm{K} \cdot \mathrm{O}$ designed the experiments, performed cell line and in vitro studies, and wrote the manuscript. M.A and Y.O. developed computational simulation methods and supervised the simulations, and wrote the manuscript. B.M and R. Kanada performed computational simulation. T.I performed FMO calculations. T.S., S.K., and T.O. designed the experiments, performed cell line and in vitro studies, N.Y, A.H, K.W, M.M, and M.N identified patients and obtained repeat biopsy samples, and analyzed clinical data. K.T. established the patient-derived cells. N.F supervised, designed the experiments, and wrote the manuscript. R. Katayama designed the study, performed cell line, patient derived cell, and in vitro and in vivo experiments; supervised the experiments; and wrote the manuscript.

\section{Appendix A. Supplementary data}

Supplementary data to this article can be found online at https://doi. org/10.1016/j.ebiom.2019.01.019. 


\section{References}

[1] Soda M, Choi YL, Enomoto M, Takada S, Yamashita Y, Ishikawa S, et al. Identification of the transforming EML4-ALK fusion gene in non-small-cell lung cancer. Nature 2007;448(7153):561-6.

[2] Kohno T, Nakaoku T, Tsuta K, Tsuchihara K, Matsumoto S, Yoh K, et al. Beyond ALKRET, ROS1 and other oncogene fusions in lung cancer. Transl Lung Cancer Res 2015;4 (2):156-64.

[3] Hallberg B, Palmer RH. Mechanistic insight into ALK receptor tyrosine kinase in human cancer biology. Nat Rev Cancer 2013;13(10):685-700.

[4] Zhang G, Scarborough H, Kim J, Rozhok AI, Chen YA, Zhang X, et al. Coupling an EML4-ALK-centric interactome with RNA interference identifies sensitizers to ALK inhibitors. Sci Signal 2016;9(450):rs12.

[5] Shaw AT, Kim DW, Nakagawa K, Seto T, Crino L, Ahn MJ, et al. Crizotinib versus chemotherapy in advanced ALK-positive lung cancer. N Engl J Med 2013;368(25): 2385-94.

[6] Solomon BJ, Mok T, Kim DW, Wu YL, Nakagawa K, Mekhail T, et al. First-line crizotinib versus chemotherapy in ALK-positive lung cancer. N Engl J Med 2014;371 (23):2167-77.

[7] Peters S, Camidge DR, Shaw AT, Gadgeel S, Ahn JS, Kim DW, et al. Alectinib versus crizotinib in untreated ALK-positive non-small-cell lung cancer. N Engl J Med 2017;377(9):829-38

[8] Hida T, Nokihara H, Kondo M, Kim YH, Azuma K, Seto T, et al. Alectinib versus crizotinib in patients with ALK-positive non-small-cell lung cancer (J-ALEX): an openlabel, randomised phase 3 trial. Lancet 2017;390(10089):29-39.

[9] Gainor JF, Dardaei L, Yoda S, Friboulet L, Leshchiner I, Katayama R, et al. Molecular mechanisms of resistance to first- and second-generation ALK inhibitors in ALKrearranged lung cancer Cancer Discov 2016:6(10):1118-33.

[10] Ou SH, Greenbowe J, Khan ZU, Azada MC, Ross JS, Stevens PJ, et al. I1171 missense mutation (particularly $I 1171 \mathrm{~N}$ ) is a common resistance mutation in ALK-positive NSCLC patients who have progressive disease while on alectinib and is sensitive to ceritinib. Lung Cancer 2015;88(2):231-4.

[11] Zou HY, Friboulet L, Kodack DP, Engstrom LD, Li Q West M, et al. PF-06463922, an ALK/ROS1 inhibitor, overcomes resistance to first and second generation ALK inhibitors in preclinical models. Cancer Cell 2015;28(1):70-81.

[12] Yoda S, Lin JJ, Lawrence MS, Burke BJ, Friboulet L, Langenbucher A, et al. Sequentia ALK inhibitors can select for lorlatinib-resistant compound ALK mutations in ALKpositive lung cancer. Cancer Discov 2018;8(6):714-29.

[13] Shaw AT, Friboulet L, Leshchiner I, Gainor JF, Bergqvist S, Brooun A, et al. Resensitization to crizotinib by the lorlatinib ALK resistance mutation L1198F. N Engl J Med 2016;374(1):54-61.

[14] Fujitani H, Tanida Y, Matsuura A. Massively parallel computation of absolute binding free energy with well-equilibrated states. Phys Rev E Stat Nonlinear Soft Matter Phys 2009;79(2 Pt 1):021914.

[15] Kitaura K, Sawai T, Asada T, Nakano T, Uebayasi M. Pair interaction molecular orbital method: an approximate computational method for molecular interactions. Chem Phys Lett 1999;312(2):319-24.

[16] Goto J, Kataoka R, Muta H, Hirayama N. ASEDock-docking based on alpha spheres and excluded volumes. J Chem Inf Model 2008;48(3):583-90.

[17] Schmidt MW, Baldridge KK, Boatz JA, Elbert ST, Gordon MS, Jensen JH, et al. General atomic and molecular electronic structure system. J Comput Chem 1993;14(11): 1347-63.

[18] Bayly CI, Cieplak P, Cornell W, Kollman PA. A well-behaved electrostatic potential based method using charge restraints for deriving atomic charges: the RESP model. J Phys Chem 1993;97(40):10269-80.

[19] Wang J, Wolf RM, Caldwell JW, Kollman PA, Case DA. Development and testing of a general amber force field. J Comput Chem 2004;25(9):1157-74.

[20] Wang J, Cieplak P, Kollman PA. How well does a restrained electrostatic potential (RESP) model perform in calculating conformational energies of organic and biological molecules. J Comput Chem 2000;21(12):1049-74.
[21] Jorgensen WL, Chandrasekhar J, Madura JD, Impey RW, Klein ML. Comparison of simple potential functions for simulating liquid water. J Chem Phys 1983;79(2): 926-35.

[22] Hess B, Kutzner C, van der Spoel D, Lindahl E. GROMACS 4: algorithms for highly efficient, load-balanced, and scalable molecular simulation. J Chem Theory Comput 2008;4(3):435-47.

[23] Araki M, Kamiya N, Sato M, Nakatsui M, Hirokawa T, Okuno Y. The effect of conformational flexibility on binding free energy estimation between kinases and their inhibitors. J Chem Inf Model 2016;56(12):2445-56.

[24] Fujitani H, Tanida Y, Matsuura A. Massively parallel computation of absolute binding free energy with well-equilibrated states. Phys Rev E 2009;79(2):021914.

[25] Ishikawa T, Kuwata K. Fragment molecular orbital calculation using the RI-MP2 method. Chem Phys Lett 2009;474:195-8.

[26] Dunning T. Gaussian basis sets for use in correlated molecular calculations. I. The atoms boron through neon and hydrogen. J Chem Phys 1989;90:1007-23.

[27] Ishikawa T, Ishikura T, Kuwata K. Theoretical study of the prion protein based on the fragment molecular orbital method. J Comput Chem 2009;30(16):2594-601.

[28] Katayama R, Kobayashi Y, Friboulet L, Lockerman EL, Koike S, Shaw AT, et al. Cabozantinib overcomes crizotinib resistance in ROS1 fusion-positive cancer. Clin Cancer Res 2015;21(1):166-74.

[29] Zhang S, Anjum R, Squillace R, Nadworny S, Zhou T, Keats J, et al. The potent ALK inhibitor brigatinib (AP26113) overcomes mechanisms of resistance to first- and second-generation ALK inhibitors in preclinical models. Clin Cancer Res 2016;22 (22):5527-38.

[30] Huang WS, Liu S, Zou D, Thomas M, Wang Y, Zhou T, et al. Discovery of brigatinib (AP26113), a phosphine oxide-containing, potent, orally active inhibitor of anaplastic lymphoma kinase. J Med Chem 2016;59(10):4948-64.

[31] Richards MW, Law EW, Rennalls LP, Busacca S, O'Regan L, Fry AM, et al. Crystal structure of EML1 reveals the basis for Hsp90 dependence of oncogenic EML4-ALK by disruption of an atypical beta-propeller domain. Proc Natl Acad Sci U S A 2014;111(14): 5195-200

[32] Katayama R, Khan TM, Benes C, Lifshits E, Ebi H, Rivera VM, et al. Therapeutic strategies to overcome crizotinib resistance in non-small cell lung cancers harboring the fusion oncogene EML4-ALK. Proc Natl Acad Sci U S A 2011;108(18):7535-40.

[33] Li J, Sun R, Wu Y, Song M, Li J, Yang Q, et al. L1198F mutation resensitizes crizotinib to ALK by altering the conformation of inhibitor and ATP binding sites. Int J Mol Sci 2017;18(3).

[34] Roskoski Jr R. Anaplastic lymphoma kinase (ALK) inhibitors in the treatment of ALKdriven lung cancers. Pharmacol Res 2017; 117:343-56.

[35] Sun HY, Ji FQ. A molecular dynamics investigation on the crizotinib resistance mechanism of C1156Y mutation in ALK. Biochem Biophys Res Commun 2012;423(2): 319-24.

[36] Kaur G, Gazit A, Levitzki A, Stowe E, Cooney DA, Sausville EA. Tyrphostin induced growth inhibition: correlation with effect on p210bcr-abl autokinase activity in K562 chronic myelogenous leukemia. Anticancer Drugs 1994;5(2):213-22.

[37] Kaur G, Sausville EA. Altered physical state of p210bcr-abl in tyrphostin AG957treated K562 cells. Anticancer Drugs 1996;7(8):815-24.

[38] Chandra J, Tracy J, Loegering D, Flatten K, Verstovsek S, Beran M, et al. Adaphostininduced oxidative stress overcomes BCR/ABL mutation-dependent and -independent imatinib resistance. Blood 2006;107(6):2501-6.

[39] Bauer DC, McMorran BJ, Foote SJ, Burgio G. Genome-wide analysis of chemically induced mutations in mouse in phenotype-driven screens. BMC Genomics 2015;16: 866.

[40] Kogita A, Togashi Y, Hayashi H, Banno E, Terashima M, De Velasco MA, et al. Activated MET acts as a salvage signal after treatment with alectinib, a selective ALK inhibitor, in ALK-positive non-small cell lung cancer. Int J Oncol 2015;46(3):1025-30.

[41] Dong X. Fernandez-Salas E, Li E, Wang S. Elucidation of resistance mechanisms to second-generation ALK inhibitors alectinib and ceritinib in non-small cell lung cancer cells. Neoplasia 2016;18(3):162-71. 